\title{
Signatures of Fractionalization in Spin Liquids from Interlayer Thermal Transport
}

\author{
Yochai Werman, ${ }^{1}$ Shubhayu Chatterjee, ${ }^{2}$ Siddhardh C. Morampudi, ${ }^{3}$ and Erez Berg ${ }^{1,4}$ \\ ${ }^{1}$ Department of Condensed Matter Physics, Weizmann Institute of Science, Rehovot 76100, Israel \\ ${ }^{2}$ Department of Physics, Harvard University, Cambridge, Massachusetts 02138, USA \\ ${ }^{3}$ Department of Physics, Boston University, Boston, Massachusetts 02215, USA \\ ${ }^{4}$ James Frank Institute and the Department of Physics, University of Chicago, \\ Chicago, Illinois 60637, USA
}

\begin{abstract}
(Received 15 August 2017; revised manuscript received 23 April 2018; published 11 September 2018)
Quantum spin liquids (QSLs) are intriguing phases of matter possessing fractionalized excitations. Several quasi-two-dimensional materials have been proposed as candidate QSLs, but direct evidence for fractionalization in these systems is still lacking. In this paper, we show that the interplane thermal conductivity in layered QSLs carries a unique signature of fractionalization. We examine several types of gapless QSL phases - a $Z_{2}$ QSL with either a Dirac spectrum or a spinon Fermi surface, and a $U(1)$ QSL with a Fermi surface - and consider both clean and disordered systems. In all cases, the in-plane and $c$-axis thermal conductivities have a different power-law dependence on temperature because of the different mechanisms of transport in the two directions: In the planes, the thermal current is carried by fractionalized excitations, whereas the interplane current is carried by integer (nonfractional) excitations. In layered $Z_{2}$ and $U(1)$ QSLs with a Fermi surface, and in the disordered $Z_{2}$ QSL with a Dirac dispersion, the $c$-axis thermal conductivity is parametrically smaller than the in-plane one but parametrically larger than the phonon contribution at low temperatures.
\end{abstract}

DOI: 10.1103/PhysRevX.8.031064

Subject Areas: Condensed Matter Physics

\section{INTRODUCTION}

Quantum spin liquids (QSLs) are phases of matter with intrinsic topological order, which cannot be characterized by local order parameters as typically used in symmetrybreaking phases. Instead, their primary characteristic is the emergence of excitations with fractional quantum numbers [1-6]. The presence of these excitations is related to the existence of long-range entanglement in ground states of such systems $[7,8]$. In addition, the excitations are accompanied by an emergent gauge field leading to a low-energy description in terms of gauge theories. The relevant gauge group can be discrete (e.g., $\mathbb{Z}_{2}$ ) or continuous [e.g., $\left.U(1)\right]$. The matter excitation spectrum may be gapped (as in a gapped $Z_{2}$ phase [9-15]) or gapless [as in a gapless $Z_{2}$ [16,17] or $U(1)$ [18-26] QSL].

Several materials have been proposed as candidates for spin liquids; these three-dimensional materials are often layered compounds of frustrated 2D lattices, such as kagome and triangular lattices. For example, members of the iridate family [27-30] have been proposed to display

Published by the American Physical Society under the terms of the Creative Commons Attribution 4.0 International license. Further distribution of this work must maintain attribution to the author(s) and the published article's title, journal citation, and DOI.
QSL gapless $Z_{2}$ behavior; the triangular organic salt $\mathrm{EtMe}_{3} \mathrm{Sb}\left[\mathrm{Pd}(\mathrm{dmit})_{2}\right]_{2}$ has been proposed to have a spinon Fermi surface, while $\kappa-(\mathrm{ET})_{2} \mathrm{Cu}_{2}(\mathrm{CN})_{3}$ is believed to be a gapped QSL [31-34]. In addition, the material Herbertsmithite is thought to be either a gapless or a small gap QSL, with its class not yet known [35-46].

The excitations of QSLs can carry fractional quantum numbers corresponding to global symmetries possessed by the system $[13,47,48]$ and also possess fractional (anyonic) statistics $[10,48-50]$. There have been numerous proposals to detect these fractional quantum numbers and statistics in QSL materials [48,51-57]. The presence of fractionalization itself has primarily been deduced through a diffuse scattered intensity seen in inelastic neutron scattering experiments on various candidate spin liquids at temperatures much smaller than the relevant exchange coupling [43,58]. The absence of sharp features in the neutron scattering intensity is attributed to the presence of a multiparticle continuum [59]. However, such broadening can also arise from other factors such as disorder, and it would be useful to have additional signatures of fractionalization.

In this work, we propose the interplane thermal conductivity $\kappa_{c}$ as a probe for fractionalization in a system of weakly coupled layers of two-dimensional gapless QSLs [60]. The in-layer thermal conductivity $\kappa_{a b}$ in these materials is dominated by the low-energy fractionalized 
excitations pertinent to the type of QSL in question; in contrast, the thermal current between the planes must be carried by a gauge-invariant excitation with integer quantum numbers. This is because the emergent gauge charge carried by fractionalized excitations is conserved separately in each layer, and therefore, a single spinon cannot move from one layer to the next. Moreover, a non-gauge-invariant fractionalized excitation, such as a spinon, is highly nonlocal in space (it is composed of a long "string" of local spin operators). This implies that the matrix element of a local operator to transfer pairs of spinons from one layer to another decays exponentially with the spatial separation between the two spinons. Therefore, only pairs of nearby spinons can hop between adjacent layers.

The situation is depicted schematically in Fig. 1, where a single spinon is deconfined and may propagate freely in each plane, while only pairs of spinons may hop between planes. Therefore, $\kappa_{c}$ in a gapless QSL is expected to be qualitatively different from the in-layer thermal

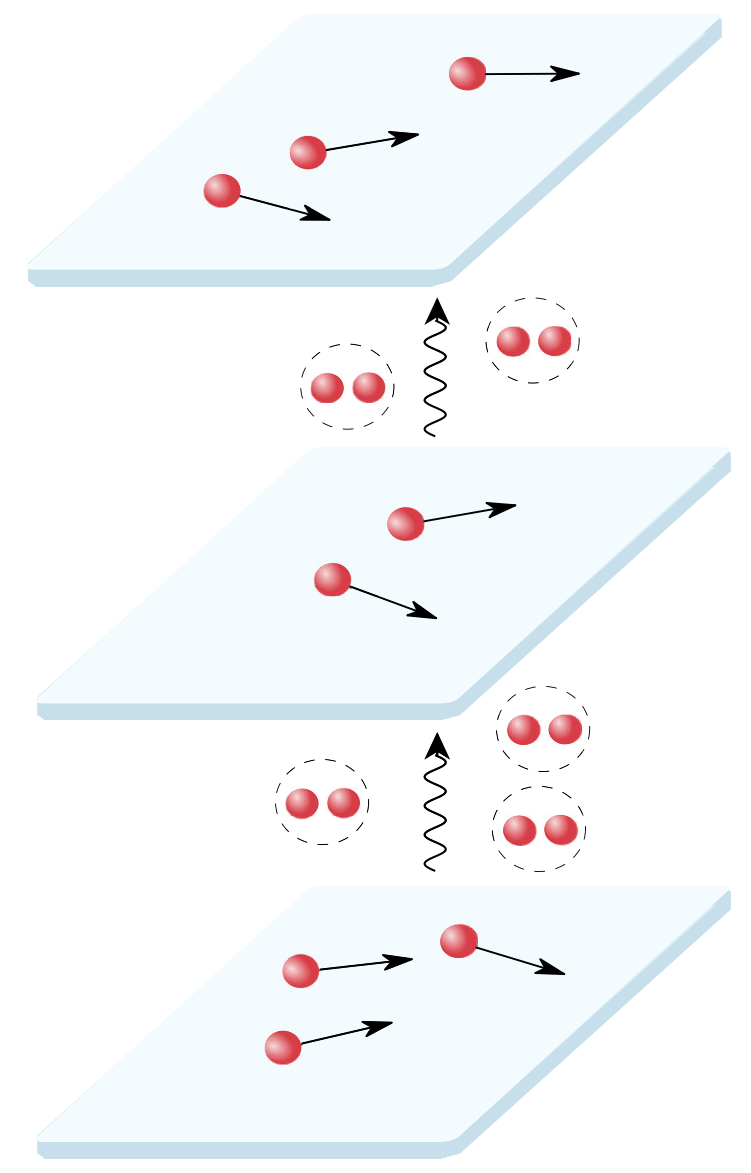

FIG. 1. A schematic representation of the difference between in-plane and interplane transport. In each QSL plane, the spinons are deconfined and may travel freely. However, transport between the planes is only possible via gauge-invariant excitations, such as spinon pairs. conductivity and to obey a different power law at low temperatures [61]. An experimental detection of such a parametrically large anisotropy in the ratio $\kappa_{a b} / \kappa_{c}$ at low temperatures will be a strong indication of the existence of fractionalized excitations and hence a QSL state.

Our findings are summarized in Table I. We have considered three cases: a gapless $Z_{2}$ QSL with either a Dirac spectrum or a spinon Fermi surface, and $U(1)$ QSL with a spinon Fermi surface. In all cases, the inplane and $c$-axis thermal conductivity follow qualitatively different behavior as a function of temperature, for both clean and mildly disordered systems. In all QSLs we consider, the interplane thermal conductivity follows a power-law behavior in temperature, with an exponent that is larger than for the corresponding intraplane behavior. Interestingly, in some cases, the exponent of the interplane thermal conductivity is smaller than 3, and therefore, it is parametrically larger than the phonon contribution (proportional to $T^{3}$ ) at sufficiently low temperatures.

\section{CLEAN $Z_{2}$ QUANTUM SPIN LIQUID}

We begin by considering a layered system where each layer forms a $Z_{2}$ QSL with gapless fermionic excitations. The fermions may either have a Dirac spectrum or form a Fermi surface. As a concrete example of the gapless $Z_{2}$ QSL, one may consider the gapless phase of the Kitaev honeycomb model [16], which consists of spin-1/2 s interacting in an anisotropic manner on a two-dimensional hexagon lattice. We use this model to facilitate our discussion; our conclusions are generic to any gapless $Z_{2}$ QSL.

The low-energy theory of the Kitaev QSL phase may be described either as two linearly dispersing Majorana fermions or, equivalently, as a single complex Dirac theory. Here, we consider a three-dimensional layered generalization of the Kitaev model. The low-energy effective Hamiltonian of each layer is given by

TABLE I. In-plane and $c$-axis thermal conductivity for several types of QSL. Here, $Z_{2}$ Dirac refers to a $Z_{2}$ QSL with a Dirac spectrum of fermionic fractional excitations, and $Z_{2}$ FS is a $Z_{2}$ QSL with a Fermi surface of fractional excitations. Note that $U(1)$ refers to a spinon Fermi surface coupled to a $U(1)$ fluctuating gauge field, and $\alpha=6 \Delta_{A} /\left(\pi+\Delta_{A}\right)$, with $\Delta_{A}$ the (dimensionless) time-reversal (TR) preserving disorder strength [see Eq. (11)]. The result for the clean $Z_{2}$ FS case is correct up to logarithmic factors.

\begin{tabular}{llcllc}
\hline \hline & \multicolumn{2}{c}{ In-plane } & & \multicolumn{2}{c}{$c$-axis } \\
\cline { 2 - 3 } \cline { 5 - 6 } & Clean & Disordered & & Clean & Disordered \\
\hline$Z_{2}$ Dirac & $T[62]$ & $T$ & & $T^{5}$ & $T^{5-\alpha}$ \\
$Z_{2}$ FS & $T^{-1}$ & $T$ & & $T^{3}$ & $T^{2}$ \\
$U(1)$ & $T^{1 / 3}[63]$ & $T[63]$ & & $T^{5 / 3}$ & $T^{2}$ \\
\hline \hline
\end{tabular}


$H_{l}^{Z_{2}}=\int \frac{d^{2} k}{(2 \pi)^{2}} \psi_{l}^{\dagger}(\mathbf{k})\left[v \boldsymbol{\sigma} \cdot \mathbf{k}+m \sigma^{z}+\Delta \sigma^{0}\right] \psi_{l}(\mathbf{k})$,

where $l$ is the layer index, and $\psi_{l}^{\dagger}(\mathbf{k})=\left(\psi_{l}^{A \dagger}(\mathbf{k})\right.$, $\left.\psi_{l}^{B^{\dagger}}(\mathbf{k})\right)$ is a spinor of complex fermionic spinon creation operators in layer $l$, with $A, B$ denoting the sublattice. Here, $\boldsymbol{\sigma}$ is a vector of Pauli matrices, $\mathbf{k}=\left(k_{x}, k_{y}\right)$ is measured relative to the corner of the honeycomb lattice (the $\mathbf{K}$ point), and $v$ is the Fermi velocity. Note that $m$ and $\Delta$ describe a mass gap and an effective chemical potential, respectively. Throughout the paper, we have set $\hbar=1$. In Appendix A, we show an explicit microscopic spin Hamiltonian that leads to a low-energy effective Hamiltonian in Eq. (1). The $m$ and $\Delta$ terms arise from
TR-breaking three-spin interactions. On the honeycomb lattice, in the presence of time reversal (TR) symmetry, $m=\Delta=0$, and the Fermi energy is at the Dirac point. Breaking TR symmetry [17], or considering generalizations of the Kitaev model to other lattices [64-69], allows for a stable Fermi surface. In all $Z_{2}$ QSLs we consider, the fermionic excitations ("spinons") are gapless, which corresponds to $|\Delta| \geq|m|$. The fluxes of the $Z_{2}$ gauge field ("visons") are gapped.

Although the in-plane theory is described by fractional excitations, interplane transport must be mediated by gauge-invariant excitations. The most relevant interlayer coupling terms that are allowed by symmetry are given by

$$
\begin{aligned}
H_{\perp}^{Z_{2}}= & J_{\perp} \sum_{\left\langle l, l^{\prime}\right\rangle} \sum_{\alpha=0,1,2,3} F_{\alpha} \int \frac{d^{2} k}{(2 \pi)^{2}} \frac{d^{2} k^{\prime}}{(2 \pi)^{2}} \frac{d^{2} q}{(2 \pi)^{2}} \psi_{l}^{\dagger}(\mathbf{k}) \sigma^{\alpha} \psi_{l}(\mathbf{k}+\mathbf{q}) \psi_{l^{\prime}}^{\dagger}\left(\mathbf{k}^{\prime}\right) \sigma^{\alpha} \psi_{l^{\prime}}\left(\mathbf{k}^{\prime}-\mathbf{q}\right) \\
& +J_{\perp} \sum_{\left\langle l, l^{\prime}\right\rangle} F_{4} \int \frac{d^{2} k}{(2 \pi)^{2}} \frac{d^{2} k^{\prime}}{(2 \pi)^{2}} \frac{d^{2} q}{(2 \pi)^{2}}\left[\psi_{l}^{A \dagger}(\mathbf{k}) \psi_{l}^{B^{\dagger}}(-\mathbf{k}-\mathbf{q}) \psi_{l^{\prime}}^{A}\left(\mathbf{k}^{\prime}\right) \psi_{l^{\prime}}^{B}\left(\mathbf{k}^{\prime}-\mathbf{q}\right)+\text { H.c. }\right],
\end{aligned}
$$

where $\left\langle l, l^{\prime}\right\rangle$ are neighboring layers, $\sigma^{\alpha}$ are the Pauli matrices (with $\sigma^{0}$ the identity matrix), $J_{\perp}$ is the strength of the interplane coupling, and $F_{0, \ldots, 4}$ are dimensionless coupling constants. In Appendix. A4, we argue that, generically, $J_{\perp}$ is proportional to the microscopic spinspin interlayer interactions.

For simplicity, we mostly focus on the case where only $F_{0}=F$ is nonzero. A derivation of such a coupling term from a microscopic spin-spin interaction is given in Appendix A. We believe that the particular form of the interlayer coupling is not important; the contribution to the thermal conductivity from other terms gives the same parametric dependence on temperature. The crucial point is that the interplane coupling term must contain an even number of fermion operators from each layer, as a single fractional excitation may not hop from one layer to another.

In a generic $Z_{2}$ QSL, there are also short-range intraplane interactions between the fermionic spinons. However, for most of the following discussion, we can ignore such interactions, as they are irrelevant in the Dirac case and lead to a Landau Fermi liquid state with well-defined quasiparticles in the Fermi surface case.

For a clean $Z_{2}$ QSL, whose low-energy theory is described by weakly interacting fermions, the interlayer thermal conductivity may be calculated to lowest order in $J_{\perp}$ using Fermi's golden rule. We work in the basis of the eigenvalues of the in-plane Hamiltonian; we therefore revert from the sublattice $(\alpha=A, B)$ to the band $(\lambda= \pm 1)$ basis and consider the transformed function $F_{\mathbf{k}, \mathbf{k}^{\prime}, \mathbf{q}}^{\lambda_{1} \ldots \lambda_{4}}$ in this basis. In the case of a Dirac spectrum, the eigenstates of the in-plane Hamiltonian are given by $a_{l}^{\lambda= \pm}(\mathbf{k})=\left[\psi_{l}^{A}(\mathbf{k}) \pm e^{i \phi_{\mathbf{k}}} \psi_{l}^{B}(\mathbf{k})\right] / \sqrt{2}$, with energy $\epsilon_{\mathbf{k}}^{\lambda}=$ $\lambda v k ;$ here, $\phi_{\mathbf{k}}=\operatorname{atan}\left(k_{y} / k_{x}\right)$. In this basis, $F_{\mathbf{k}, \mathbf{k}^{\prime}, \mathbf{q}}^{\lambda_{1} \ldots \lambda_{4}}=$ $\frac{1}{4}\left[1+\lambda_{1} \lambda_{2} e^{i\left(\phi_{\mathbf{k}}-\phi_{\mathbf{k}+\mathbf{q}}\right)}\right]\left[1+\lambda_{3} \lambda_{4} e^{i\left(\phi_{\mathbf{k}^{\prime}}-\phi_{\mathbf{k}^{\prime}-\mathbf{q}}\right)}\right] . \quad$ Energy is transported between layers by the excitation of spinon pairs; thus, if a temperature difference $\delta T$ is applied between two adjacent layers $l$ and $l^{\prime}$, the rate with which energy transfer occurs, for the specific momenta $\mathbf{k}, \mathbf{k}+\mathbf{q}$, $\mathbf{k}^{\prime}, \mathbf{k}^{\prime}-\mathbf{q}$, is

$$
\begin{aligned}
\Gamma_{\mathbf{k}, \mathbf{k}+\mathbf{q}, \mathbf{k}^{\prime}, \mathbf{k}^{\prime}-\mathbf{q}}^{E}= & \frac{2 \pi}{Z} J_{\perp}^{2} \sum_{\lambda_{1 \ldots 4}}\left|F_{\mathbf{k}, \mathbf{k}^{\prime}, \mathbf{q}}^{\lambda_{1} \ldots \lambda_{4}}\right|^{2} \sum_{i_{l}, i_{l^{\prime}} ; f_{l}, f_{l^{\prime}}}\left[\exp \left(-\frac{E_{i, l}}{T+\delta T}-\frac{E_{i, l^{\prime}}}{T}\right)-\exp \left(-\frac{E_{f, l}}{T+\delta T}-\frac{E_{f, l^{\prime}}}{T}\right)\right] \\
& \times\left|\left\langle f_{l}\left|\left\langle f_{l^{\prime}}\left|a_{l}^{\lambda_{1} \dagger}(\mathbf{k}) a_{l}^{\lambda_{2}}(\mathbf{k}+\mathbf{q}) a_{l^{\prime}}^{\lambda_{3} \dagger}\left(\mathbf{k}^{\prime}\right) a_{l^{\prime}}^{\lambda_{4}}\left(\mathbf{k}^{\prime}-\mathbf{q}\right)\right| i_{l}\right\rangle\right| i_{l^{\prime}}\right\rangle\right|^{2}\left(E_{i, l}-E_{f, l}\right) \delta\left(E_{i, l}+E_{i, l^{\prime}}-E_{f, l}-E_{f, l^{\prime}}\right),
\end{aligned}
$$

where $\left|i_{l}\right\rangle,\left|f_{l}\right\rangle$ are the initial and final many-body states of layer $l$ (which are eigenstates of the $J_{\perp}=0$ Hamiltonian), with energies $E_{i, l}$ and $E_{f, l}$, respectively, and similarly for layer $l^{\prime}$. Here, $Z$ is the partition function. 
The thermal conductivity is then given by (here, $J^{Q}$ is the thermal current)

$$
\begin{aligned}
\kappa_{c}= & \frac{\partial J^{Q}}{\partial \delta T}=\int \frac{d^{2} k}{(2 \pi)^{2}} \frac{d^{2} k^{\prime}}{(2 \pi)^{2}} \frac{d^{2} q}{(2 \pi)^{2}} \frac{\partial}{\partial \delta T} \Gamma_{\mathbf{k}, \mathbf{k}+\mathbf{q}, \mathbf{k}^{\prime}, \mathbf{k}^{\prime}-\mathbf{q}}^{E} \\
= & 2 \pi \frac{J_{\perp}^{2}}{T^{2}} \sum_{\lambda_{1} \ldots 4} \int d \epsilon_{1} d \epsilon_{2} d \epsilon_{3}\left(1-n_{F}\left(\epsilon_{1}\right)\right) n_{F}\left(\epsilon_{2}\right)\left(1-n_{F}\left(\epsilon_{3}\right)\right) n_{F}\left(\epsilon_{1}-\epsilon_{2}+\epsilon_{3}\right) \times\left(\epsilon_{1}-\epsilon_{2}\right)^{2} \\
& \times \int \frac{d^{2} k}{(2 \pi)^{2}} \frac{d^{2} k^{\prime}}{(2 \pi)^{2}} \frac{d^{2} q}{(2 \pi)^{2}}\left|F_{\mathbf{k}, \mathbf{k}^{\prime}, \mathbf{q}}^{\lambda_{1} \ldots \lambda_{4}}\right|^{2} \delta\left(\epsilon_{1}-\epsilon_{\mathbf{k}}^{\lambda_{1}}\right) \delta\left(\epsilon_{2}-\epsilon_{\mathbf{k}+\mathbf{q}}^{\lambda_{2}}\right) \delta\left(\epsilon_{3}-\epsilon_{\mathbf{k}^{\prime}}^{\lambda_{3}}\right) \delta\left(\epsilon_{1}+\epsilon_{3}-\epsilon_{2}-\epsilon_{\mathbf{k}^{\prime}-\mathbf{q}}^{\lambda_{4}}\right),
\end{aligned}
$$

where $n_{F}(\epsilon)$ is the Fermi function.

For the case of a $Z_{2}$ QSL with a Dirac spectrum, the dependence of the integral on temperature can be evaluated easily by rescaling $\epsilon \Rightarrow \epsilon / T$ and $\left\{\mathbf{k}, \mathbf{k}^{\prime}, \mathbf{q}\right\} \Rightarrow$ $\left\{\mathbf{k} / v T, \mathbf{k}^{\prime} / v T, \mathbf{q} / v T\right\}$. This gives the result

$$
\kappa_{c} \sim \frac{J_{\perp}^{2}}{v^{6}} T^{5} \quad \text { (clean } Z_{2} \text { with Dirac spectrum). }
$$

The case of a $Z_{2}$ QSL with a Fermi surface corresponds to $\Delta \neq 0$ in Eq. (1). To simplify the calculation, we set the mass term in Eq. (1) such that $\Delta>m$ but $|\Delta-m| \ll m$. In this limit, the eigenstates of the band that crosses the Fermi energy simplify to $a(\mathbf{k})=\psi^{A}(\mathbf{k})$, with a nonrelativistic dispersion $\epsilon_{\mathbf{k}}=k^{2} / 2 m^{*}-\mu$, with $\mu=\Delta-m$ and $m^{*}=m / v^{2}$. The result should not depend on this choice.

The evaluation of the integrals in Eq. (4) for the case of a Fermi surface is described in Appendix B. After integrating over $\mathbf{k}, \mathbf{k}^{\prime}$, and $\epsilon_{1,2,3}, \kappa_{c}$ has the form

$$
\begin{aligned}
\kappa_{c} & \sim J_{\perp}^{2} T^{3} \int \frac{d^{2} k d^{2} k^{\prime} d^{2} q}{(2 \pi)^{6}} \delta\left(\epsilon_{\mathbf{k}}\right) \delta\left(\epsilon_{\mathbf{k}+\mathbf{q}}\right) \delta\left(\epsilon_{\mathbf{k}^{\prime}}\right) \delta\left(\epsilon_{\mathbf{k}^{\prime}-\mathbf{q}}\right) \\
& \sim J_{\perp}^{2} \frac{\nu^{4}}{k_{F}^{2}} T^{3} \int_{0}^{2 k_{F}} d q \frac{1}{q} \frac{1}{1-q^{2} / 4 k_{F}^{2}},
\end{aligned}
$$

where $\nu=m^{*} / 2 \pi$ is the density of states on the Fermi energy, and $k_{F}=\sqrt{2 m^{*} \mu}$. This integral is logarithmically divergent; this is similar to the divergence of the electronic self-energy in a Fermi liquid in two dimensions [70]. As in a Fermi liquid, intralayer short-range interactions between the spinons lead to a finite spinon lifetime $\tau \propto 1 / T^{2}$. The associated broadening of the spinon spectral function provides an infrared cutoff for the logarithm [71], giving

$$
\kappa_{c} \sim J_{\perp}^{2} \frac{\nu^{4}}{k_{F}^{2}} T^{3} \log (\Lambda / T) \quad\left(\text { clean } Z_{2} \text { with FS }\right)
$$

with $\Lambda$ a high-energy cutoff, of the order of the Fermi energy (which is proportional to the exchange coupling between the original spins).

The in-plane thermal conductivity of the $Z_{2}$ QSL with a Fermi surface is given, using the Einstein relation, by $\kappa_{a b} \sim c_{V} v_{F}^{2} \tau / 2$, where $c_{V}=\pi^{2} \nu T / 3$ is the specific heat of the system at low temperatures, $v_{F}=k_{F} / m^{*}$ is the Fermi velocity, and $\tau$ is the spinon lifetime. In a perfectly clean crystal, the finite lifetime is due to the weak short-range interaction between the spinons mediated by the gapped gauge field (assuming that Umklapp processes are available to relax the total momentum of the scattering spinons). The lifetime is given by $\tau^{-1} \sim T^{2} \log (\Lambda / T)$ as discussed earlier, and therefore, we have

$$
\kappa_{a b} \sim[T \log (\Lambda / T)]^{-1} .
$$

\section{DISORDERED $Z_{2}$ QUANTUM SPIN LIQUID}

As we now show, quenched disorder changes the lowtemperature interplane transport in a qualitative way. The effects of disorder depend crucially on the type of disorder, which is subject to the symmetry of the problem. Consider, for example, the case of the honeycomb Kitaev model with time-reversal symmetry. Then, disorder can take the form of a random bond strength that translates to a random vector potential [72] in the low-energy Dirac Hamiltonian, Eq. (1). Breaking time-reversal symmetry can induce random scalar potential and mass terms as well (see Appendix $\mathrm{C} 1$ for a demonstration of how such terms arise in a disordered version of the Kitaev model).

Here, we focus on random vector and scalar potentials; a random mass term is important at the transition between different gapped spin-liquid states, a case we will not consider in the present work. The disordered part of the low-energy effective Hamiltonian in layer $l$ is given by

$H_{l}^{\mathrm{dis}}=\int \frac{d^{2} k d^{2} k^{\prime}}{(2 \pi)^{4}} \psi_{l}^{\dagger}(\mathbf{k})\left(\mathcal{V}_{\mathbf{k}-\mathbf{k}^{\prime}}+v \mathcal{A}_{\mathbf{k}-k^{\prime}} \cdot \sigma\right) \psi_{l}\left(\mathbf{k}^{\prime}\right)$,

where $\mathcal{V}_{\mathbf{k}-\mathbf{k}^{\prime}}$ and $\mathcal{A}_{\mathbf{k}-\mathbf{k}^{\prime}}$ are random scalar and vector potentials, respectively. We assume that the disordered potentials in different layers are statistically independent.

First, we study the case of a Dirac QSL with timereversal symmetry, in which only a random vector potential term is allowed, $\mathcal{V}_{\mathbf{k}-\mathbf{k}^{\prime}}=0$. The effects of a vector potential disorder on a system with a Dirac dispersion were studied extensively in Ref. [73], where it was shown that such a 
term leads to a line of fixed points, characterized by scaling exponents that depend continuously on the disorder strength. Using the methods introduced in Ref. [73], we can find the scaling form of correlation functions at this fixed point, as described in detail in Appendix C2a. This allows us to show that vector potential disorder results in a modification of the exponent of the thermal conductivity, which is given by

$\kappa_{z} \sim T^{5-\alpha} \quad$ (disordered $Z_{2}$ with Dirac spectrum),

with $\alpha=6 \Delta_{A} /\left(\pi+\Delta_{A}\right), \Delta_{A}$ being the disorder strength:

$$
(2 \pi)^{2} \delta\left(\mathbf{q}+\mathbf{q}^{\prime}\right) \Delta_{A}=\left\langle\mathcal{A}_{\mathbf{q}} \mathcal{A}_{\mathbf{q}^{\prime}}\right\rangle_{\mathrm{dis}},
$$

and the average is over disorder configurations. We consider smooth disorder, such that $\psi^{\dagger} \psi^{\dagger}$ terms (corresponding to intervalley scattering in the Majorana model) are negligible.

Next, we consider the effect of disorder on a $Z_{2}$ QSL with a Fermi surface, corresponding to $\Delta \neq 0$ in Eq. (1). In this case, since time-reversal symmetry is broken, both scalar and vector disorder potentials are allowed. To simplify the computation, we neglect the vector potential in this case and assume that the scalar potential is shortrange correlated in space: $\left\langle\mathcal{V}_{\mathbf{q}} \mathcal{V}_{\mathbf{q}^{\prime}}\right\rangle_{\text {dis }}=\delta\left(\mathbf{q}+\mathbf{q}^{\prime}\right) /(2 \pi \nu \tau)$, where $\nu$ is the density of states at the Fermi level, and $\tau$ is the mean-free time of quasiparticles at the Fermi surface. Moreover, we again set the mass term in Eq. (1) such that $\Delta>m$ but $|\Delta-m| \ll m$. We expect that none of the qualitative aspects of the solution depend on these choices.

In the presence of disorder, the calculation of the thermal conductivity is most conveniently done using the Luttinger prescription [74-76]. The thermal conductivity is written as

$$
\kappa=\frac{-1}{T} \lim _{\omega \rightarrow 0} \frac{\operatorname{Im}[\Pi(\omega)]}{\omega},
$$

where $\Pi(\omega)$ is the retarded thermal current-thermal current correlation function,

$$
\Pi(\omega)=\left.\left\langle J^{Q}\left(i \omega_{n}\right) J^{Q}\left(-i \omega_{n}\right)\right\rangle\right|_{i \omega_{n} \rightarrow \omega+i \delta} .
$$

The $c$-axis thermal current operator can be derived using the energy continuity equation: $J_{c}^{Q}\left(i \omega_{n}\right)=\lim _{q_{c} \rightarrow 0} \frac{i \omega_{n} h\left(q_{c}\right)}{q_{c}}$, where $h\left(q_{c}\right)$ is the energy density operator at wave vector $q_{c}$. An explicit calculation to leading order in $J_{\perp}$ using Eq. (2) gives (see Appendixes D1 and D2)

$$
\begin{aligned}
J_{c}^{Q}\left(i \omega_{n}\right)= & \frac{1}{32} J_{\perp} F_{0} \sum_{l, \eta= \pm 1} \eta \int \frac{d^{2} k}{(2 \pi)^{2}} \frac{d^{2} k^{\prime}}{(2 \pi)^{2}} \frac{d^{2} q}{(2 \pi)^{2}} \frac{1}{\beta^{3}} \sum_{\nu_{n}, \nu_{m}, \Omega_{n}} \Omega_{n} \\
& \times \psi_{l}^{\dagger}\left(\mathbf{k}, i \nu_{n}\right) \psi_{l}\left(\mathbf{k}+\mathbf{q}, i \nu_{n}+i \Omega_{n}+i \omega_{n}\right) \\
& \times \psi_{l+\eta}^{\dagger}\left(\mathbf{k}^{\prime}, i \nu_{m}\right) \psi_{l+\eta}\left(\mathbf{k}^{\prime}-\mathbf{q}, i \nu_{m}-i \Omega_{n}\right) .
\end{aligned}
$$

Here, we have suppressed the eigenstate indices $\lambda_{1, \ldots, 4}$ since in the nonrelativistic limit $|\Delta-m| \ll m$, the wave functions of states at the Fermi surface are confined to a single sublattice. Similarly, we have suppressed the eigenstate indices in $F_{0}$, which is now momentum independent. Note that, similarly to the interplane coupling, the thermal current operator in our model is quartic in the fermionic operators, corresponding to the fact that energy is carried between the plane by the hopping of fermion pairs.

The diagrams describing the leading-order contribution to $\kappa_{c}$ are shown in Fig. 2. The computation is lengthy but straightforward, and we only describe the main steps here, deferring the details to Appendix D5. We assume that the disorder is weak, such that $k_{F} \ell \gg 1$, where $k_{F}$ is the Fermi momentum and $\ell=k_{F} \tau / m$ is the mean-free path. Under these conditions, we may use the self-consistent Born approximation [77], equivalent to summing only noncrossed diagrams [78].

A key object is the disorder-averaged four-point correlator within a single layer $\Upsilon$, depicted in Fig. 2(b):

$$
\begin{aligned}
& \Upsilon\left(\mathbf{k}, \mathbf{k}^{\prime}, \mathbf{q} ; i \nu_{n}, i \nu_{m}\right) \\
& \quad=\left\langle\psi_{l}^{\dagger}\left(\mathbf{k}, i \nu_{n}\right) \psi_{l}\left(\mathbf{k}+\mathbf{q}, i \nu_{m}\right) \psi_{l}^{\dagger}\left(\mathbf{k}^{\prime}+\mathbf{q}, i \nu_{m}\right) \psi_{l}\left(\mathbf{k}^{\prime}, i \nu_{n}\right)\right\rangle_{\mathrm{dis}} .
\end{aligned}
$$

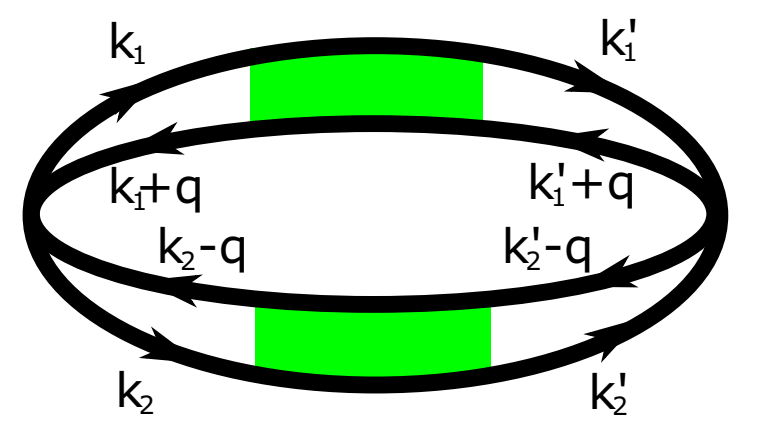

(a)

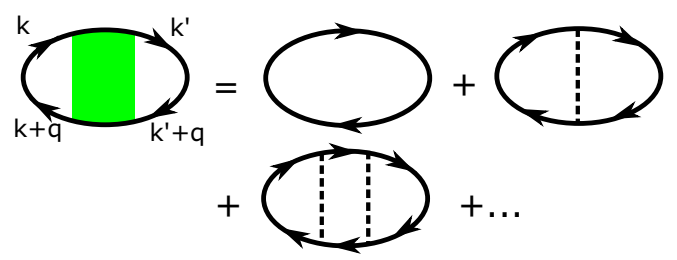

(b)

FIG. 2. (a) The thermal current-thermal current correlation diagram. The Green's functions on the top, which are functions of momentum $k_{1}$, are related to layer $l$, while those on the bottom are from layer $l^{\prime}$. Note that the current vertex consists of four Green's functions, two from each layer. (b) The disorder-averaged four-point correlator within each layer, $\Upsilon\left(\mathbf{k}, \mathbf{k}^{\prime}, \mathbf{q}\right)$. The black lines denote fully dressed fermion propagators, dashed lines represent the effects of disorder, the squiggly lines are the bare thermal current vertex, and the green area stands for the fully renormalized two-particle vertex. We work in the self-consistent Born approximation, applicable for $k_{F} \ell \gg 1$, where only ladder diagrams are taken into account. We suppress the frequency dependence for clarity. 
The thermal current correlation function, Eq. (13), is then given as a convolution of two four-point correlation functions of two adjacent layers:

$$
\begin{aligned}
\Pi\left(i \omega_{n}\right)= & \frac{1}{64} J_{\perp}^{2} \frac{1}{\beta^{3}} \sum_{\nu_{n}, \nu_{m}, \Omega_{n}} \int_{k} F_{0}^{2} \Omega_{n}^{2} \Upsilon\left(\mathbf{k}_{1}, \mathbf{k}_{1}^{\prime}, \mathbf{q} ; \nu_{n}, \nu_{n}+\Omega_{n}\right) \\
& \times \Upsilon\left(\mathbf{k}_{2}, \mathbf{k}_{2}^{\prime},-\mathbf{q} ; \nu_{m}+\omega_{n}, \nu_{m}-\Omega_{n}\right) .
\end{aligned}
$$

Here, $\int_{k}=\int\left[\left(d^{2} k_{1} d^{2} k_{2} d^{2} k_{1}^{\prime} d^{2} k_{2}^{\prime} d^{2} q\right) /(2 \pi)^{10}\right]$.

The clean, free fermion limit of this expression, with the correlator $\Upsilon\left(\mathbf{k}_{1}, \mathbf{k}_{1}{ }^{\prime}, \mathbf{q} ; i \nu_{n}, i \nu_{m}\right)=\delta\left(\mathbf{k}_{1}-\mathbf{k}_{1}{ }^{\prime}\right) G\left(\mathbf{k}_{1}, i \nu_{n}\right)$ $G\left(\mathbf{k}_{\mathbf{1}}+\mathbf{q}, i \nu_{m}\right)$, reproduces the Fermi golden rule calculation, Eq. (4). In the presence of disorder, the computation of $\Upsilon$ for small $q$ (such that $q \ell \ll 1$ ) involves a summation over a ladder series (see Appendix D5); this results in

$$
\begin{aligned}
\Upsilon\left(\mathbf{k}, \mathbf{k}^{\prime}, \mathbf{q} ; i \nu_{n}, i \nu_{m}\right) \approx & G\left(\mathbf{k}, i \nu_{n}\right) G\left(\mathbf{k}+\mathbf{q}, i \nu_{m}\right) \\
& \times\left\{\delta\left(\mathbf{k}-\mathbf{k}^{\prime}\right)+1 /\left(2 \pi \nu \tau^{2}\right)\right. \\
& \times\left[\left|\nu_{n}-\nu_{m}\right|+D \mathbf{q}^{2}\right]^{-1} G\left(\mathbf{k}^{\prime}, i \nu_{n}\right) \\
& \left.\times G\left(\mathbf{k}^{\prime}+\mathbf{q}, i \nu_{m}\right)\right\},
\end{aligned}
$$

where the diffusion constant $D=v^{2} \tau / 2$, with $\tau$ the disorder-induced single-particle lifetime, and

$$
G\left(\mathbf{k}, i \nu_{n}\right)=\frac{1}{i \nu_{n}-\epsilon_{\mathbf{k}}+i \operatorname{sign}\left(\nu_{n}\right) / 2 \tau} .
$$

Note the appearance of the diffusion kernel in Eq. (17); this is related to the diffusive behavior of the dynamical charge correlation function in a disordered system.

The computation of the sums in Eq. (16) is described in Appendix D5. The dominant contribution comes from low frequencies and momenta, where the four-point correlator takes the form (17). At low temperatures, $T<1 / \tau$, the result is

$\kappa_{c} \sim J_{\perp}^{2} \frac{\nu^{2}}{D} T^{2} \quad$ (disordered $Z_{2}$ with Fermi surface $)$.

At higher temperatures, $T \gtrsim 1 / \tau, \kappa_{c}$ crosses over to the clean form, Eq. (7). Equation (19) can also be derived from scaling arguments, assuming that the intraplane densitydensity correlation function has a diffusion form; see Appendix C2b.

In Appendix D6, we show that the $F_{4}$ pair hopping interlayer term results in the same power law, $\kappa_{c} \sim T^{2}$.

\section{IV. $U(1)$ QUANTUM SPIN LIQUID}

We further study the case of a layered $U(1)$ QSL with a spinon Fermi surface. In addition to the fermionic spinons, there exist gapless gauge field photons, which also contribute to transport. The low-energy sector of each layer is described by the Lagrangian density [20-26]
$L_{l}=\sum_{\sigma=\uparrow, \downarrow} \psi_{l, \sigma}^{\dagger}\left(\partial_{t}-i a_{0}-\mu\right) \psi_{l, \sigma}+\frac{1}{2 m} \psi_{l, \sigma}^{\dagger}(-i \nabla-\mathbf{a})^{2} \psi_{l, \sigma}$,

where $\psi_{l, \sigma}^{\dagger}$ creates a spinon at layer $l$ with spin $\sigma,\left(a_{0}, \mathbf{a}\right)$ is the $U(1)$ gauge field, $\mu$ is a chemical potential that sets the size of the spinon Fermi surface, and $m$ is the spinon effective mass. A "Maxwell" term for $a_{\nu}$, $[1 /(2 g)] \sum_{\nu \lambda} f^{\nu \lambda} f_{\nu \lambda}$, where $g$ is a coupling constant and $f_{\nu \lambda}=\partial_{\nu} a_{\lambda}-\partial_{\lambda} a_{\nu}$ is also allowed by symmetry; however, it gives rise to subleading contributions at low momenta and frequencies; hence, we drop it in the following.

Under the random phase approximation (RPA), the clean system is described by a strong-coupling fixed point, with the retarded gauge boson and spinon propagators $\left[D^{R}(\mathbf{q}, \omega)\right.$ and $G^{R}(\mathbf{k}, \omega)$, respectively] given by

$$
\begin{aligned}
D_{\alpha \beta}^{R}(\mathbf{q}, \omega) & =P_{\alpha \beta}(\mathbf{q})\left[-i \gamma \frac{\omega}{q}+\chi q^{2}\right]^{-1} \\
G^{R}(\mathbf{k}, \omega) & =\left[c(-i \omega)^{2 / 3}-\xi_{\mathbf{k}}\right]^{-1} \quad[\text { clean } U(1) \mathrm{QSL}],
\end{aligned}
$$

with $\xi_{\mathbf{k}}=k^{2} / m-\mu$ the spinon energy, $\gamma=k_{F} / \pi$, $\chi=1 /(12 \pi m), \quad c=\left(k_{F} / m\right) \chi^{-2 / 3} k_{0}^{-1 / 3}, \quad$ and $\quad P_{\alpha \beta}(\mathbf{q})=$ $\delta_{\alpha \beta}-q_{\alpha} q_{\beta} / q^{2}$, with $k_{0}$ of the order of $k_{F}=\sqrt{2 m \mu}$, the Fermi momentum. The use of the RPA has been formally justified in a large- $N$ expansion, where $N$ is the number of fermion flavors [26], but this has been shown to be problematic [79]. Additional expansion parameters have been proposed that essentially reproduce the RPA results $[80,81]$. We use the RPA approximation, assuming it is pertinent to at least some area in parameter space.

In a layered $U(1)$ QSL, heat may be transferred between the layers, both by spinon and by photon excitations. The most relevant interlayer interaction term of each sector is given by

$$
\begin{aligned}
H_{\perp}^{U(1)}= & J_{\perp}^{\mathrm{sp}} \sum_{\left\langle l, l^{\prime}\right\rangle} \int \frac{d^{2} k}{(2 \pi)^{2}} \frac{d^{2} k^{\prime}}{(2 \pi)^{2}} \frac{d^{2} q}{(2 \pi)^{2}} F_{\mathrm{sp}}^{\sigma_{1, \ldots, 4}}\left(\mathbf{k}, \mathbf{k}^{\prime}, \mathbf{q}\right) \\
& \times \psi_{l \sigma_{1}}^{\dagger}(\mathbf{k}) \psi_{l \sigma_{2}}(\mathbf{k}+\mathbf{q}) \psi_{l^{\prime} \sigma_{3}}^{\dagger}\left(\mathbf{k}^{\prime}\right) \psi_{l^{\prime} \sigma_{4}}\left(\mathbf{k}^{\prime}-\mathbf{q}\right) \\
& +J_{\perp}^{\mathrm{ph}} \int \frac{d^{2} k}{(2 \pi)^{2}} k^{2} F_{\mathrm{ph}}(\mathbf{k}) \mathbf{a}_{l}^{T}(\mathbf{k}) \mathbf{a}_{l^{\prime}}^{T}(\mathbf{k}),
\end{aligned}
$$

where $\mathbf{a}^{T}$ is the transverse part of the gauge field. The coupling functions $F_{\mathrm{sp}}$ and $F_{\mathrm{ph}}$ depend on the spatial structure of the interlayer coupling; their explicit form is unimportant. In real space, the gauge-invariant term $\nabla \times \mathbf{a}$ is related to the chirality of the underlying spin degrees of freedom [25], and therefore, the $J_{\perp}^{\mathrm{ph}}$ term corresponds to an interaction between the chiralities of the spin textures in the two layers. Microscopically, this term may be small 
compared to $J_{\perp}^{\mathrm{sp}}$ since it is of higher order in the interplane Heisenberg exchange coupling. However, as we see below, in a clean case, it gives a dominant contribution to $\kappa_{c}$ at asymptotically low temperatures.

The calculation of the spinon-mediated interplane thermal conductivity proceeds in a similar fashion as in the $Z_{2}$ QSL case. Note that $\kappa_{c}$ is given by a similar expression to Eq. (D20) (with the replacement $F \rightarrow F_{\text {sp }}$ ):

$$
\kappa_{c, \mathrm{sp}} \approx\left(J_{\perp}^{\mathrm{sp}}\right)^{2} \frac{\nu^{2}}{v^{2}} T^{3} \log \left(\frac{T}{(W / c)^{3 / 2}}\right),
$$

where $W$ is an appropriate UV cutoff (see Appendix E).

However, in the clean case, the dominant source of low$T$ thermal transport turns out to be the exchange of gauge fluctuations; this contribution may also be calculated by the Kubo formula, and it is given by (see Appendix E for details)

$$
\begin{aligned}
\kappa_{c, \mathrm{ph}} & =\frac{\left(J_{\perp}^{\mathrm{ph}}\right)^{2}}{T} \int \frac{d^{2} k}{(2 \pi)^{2}} k^{4} \int_{0}^{\infty} d \epsilon A_{\mathrm{ph}}^{2}(\mathbf{k}, \epsilon) \epsilon^{2} \partial_{\epsilon} n_{B}(\epsilon) \\
& \sim\left(J_{\perp}^{\mathrm{ph}}\right)^{2} \gamma^{2 / 3} \chi^{4 / 3} T^{5 / 3} \quad[\text { clean } U(1) \mathrm{QSL}] .
\end{aligned}
$$

Here, $A_{\mathrm{ph}}(\mathbf{k}, \epsilon)=-2 \operatorname{Im} D^{R}(\mathbf{k}, \epsilon)=\gamma\left[(|\epsilon| k) /\left(\chi^{2} k^{6}+\gamma^{2} \epsilon^{2}\right)\right]$ is the photon spectral function.

Thus, at sufficiently low temperature, $\kappa_{c, \mathrm{ph}} \gg \kappa_{c \text {,sp }}$. Note that the thermal conductivity can be written as $\kappa_{c, \mathrm{ph}} \sim T^{2-1 / z}$, where $z=3$ is the dynamical critical exponent of the fixed point described by RPA.

The introduction of disorder to the $U(1)$ theory is likely to destabilize the $z=3$ fixed point, leading instead to diffusive behavior, similar to that of a disordered Fermi liquid. In the RPA approximation, the propagators of the disordered theory are given by [82]

$$
\begin{aligned}
D_{\alpha \beta}^{R}(\mathbf{q}, \omega) & =P_{\alpha \beta}(\mathbf{q})\left[-i \omega+D q^{2}\right]^{-1}, \\
G^{R}(\mathbf{k}, \omega) & =\left[\omega-\xi_{\mathbf{k}}+i /(2 \tau)\right]^{-1} \quad[\text { disordered } U(1) \mathrm{QSL}],
\end{aligned}
$$

with $D$ a diffusion constant and $\tau$ the disorder-induced finite lifetime. The calculation of the $c$-axis thermal conductivity is then similar to the disordered $Z_{2}$ QSL case. Inserting Eq. (25) in the Kubo formula for the $c$-axis conductivity leads to

$$
\left.\kappa_{c} \propto T^{2} \quad \text { [disordered } U(1) \mathrm{QSL}\right]
$$

for both spinon and photon contributions.

\section{EXPERIMENTAL CONSIDERATIONS}

In this section, we discuss possible experimental candidate systems where thermal conductivity provides a gateway to observing QSL physics. In order to observe the magnetic contribution to the interlayer thermal conductivity, one has to be able to separate it from the phonon contribution. Since the phonon contribution scales as $T^{3}$, the magnetic contribution in certain QSLs dominates at sufficiently low temperatures. This happens in QSLs with a disordered spinon Fermi surface and in strongly disordered Dirac QSLs (see Table I). Below, we provide a rough order-of-magnitude estimate for the temperature $T_{*}$ at which the magnetic contribution exceeds the phonon one, as a function of system parameters (such as the strength of the interplane coupling, the Debye temperature, and the disorder strength). As we elaborate below, this estimate indicates that at least in some material candidates, the crossover to magnetically dominated thermal transport may occur at accessible temperatures.

We base our estimate of $T_{*}$ on the case of a QSL with a spinon FS, whose magnetic $c$-axis thermal conductivity is given by Eq. (19). We set the unit of length to be the lattice spacing $a$ and estimate $\nu \sim 1 / J, D=\frac{1}{2} v_{F} \ell_{\mathrm{sp}} \sim \frac{1}{2} J \ell_{\mathrm{sp}}$, where $J$ is the in-plane exchange coupling, and $\ell_{\text {sp }}$ is the spinon mean-free path in the plane. This gives

$$
\kappa_{\mathrm{sp}} \sim \frac{2 J_{\perp}^{2} T^{2}}{J^{3} \ell_{\mathrm{sp}}}
$$

Next, we estimate the contribution of the phonons. The acoustic phonon specific heat is $c_{V} \sim\left(T / \Theta_{D}\right)^{3}$, where $\Theta_{D}$ is the Debye frequency. The (three-dimensional) phonon diffusivity is $D_{\mathrm{ph}}=\frac{1}{3} c_{s} \ell_{\mathrm{ph}} \sim \frac{1}{3} \Theta_{D} \ell_{\mathrm{ph}}$, where $c_{s}$ is the sound velocity. Therefore, by the Einstein relation,

$$
\kappa_{\mathrm{ph}} \sim \frac{T^{3}}{3 \Theta_{D}^{2}} \ell_{\mathrm{ph}} .
$$

The temperature below which the spinon contribution to the thermal conductivity becomes larger than the phonon contribution is given by equating Eq. (27) to Eq. (28). The result is

$$
T_{*}=\frac{6 J_{\perp}^{2} \Theta_{D}^{2}}{J^{3} \ell_{\mathrm{ph}} \ell_{\mathrm{sp}}} .
$$

Note that Eq. (27) is only valid for $T \ll J$; therefore, $T_{*}$ in Eq. (29) cannot exceed $J$.

As an illustrative example, we roughly estimate the crossover temperature $T_{*}$ for kapellasite, a kagome gapless QSL candidate [83]. This is a polymorph of Herbertsmithite; however, the in-plane exchange coupling is about an order of magnitude smaller. The exchange couplings of kapellasite have been estimated from first-principle calculations [84]: $J \approx 10 \mathrm{~K}, J_{\perp} \approx 0.5 \mathrm{~K}$. We assume that kapellasite has a spinon Fermi surface and that the Debye temperature is $\Theta_{D} \sim 300 \mathrm{~K}$. The mean-free paths of the spinons and the phonons are not known. However, disorder in the planes is believed to be substantial. To get a rough estimate of the order of magnitude of $T_{*}$, let us assume a strongly disordered sample, such that $\ell_{\mathrm{sp}}=20 a$ and $\ell_{\mathrm{ph}}=200 a$. This gives 


$$
T_{*} \approx 6 \frac{0.5^{2} \times 300^{2}}{10^{3} \times 20 \times 200} \approx 35 \mathrm{mK} .
$$

Kapellasite does not order magnetically, at least down to $20 \mathrm{mK}$ [83]. Thus, for sufficiently strong disorder, we get that the crossover temperature is within experimental reach.

Let us discuss other QSL candidate materials, where the spinon contribution to $\kappa_{c}$ may be measurable. A promising candidate material is the recently discovered $2 \mathrm{D}$ spin-orbit coupled iridate $\mathrm{H}_{3} \mathrm{LiIr}_{2} \mathrm{O}_{6}$, which has been observed to be paramagnetic to very low temperatures and hosts gapless excitations $[85,86]$. Compared to other similar compounds like $\mathrm{Na}_{2} \mathrm{LiO}_{3}$ and $\mathrm{Li}_{2} \mathrm{IrO}_{3}$ (which order at low temperatures), in $\mathrm{H}_{3} \mathrm{LiIr}_{2} \mathrm{O}_{6}$ the interlayer distance is smaller due to replacement of $\mathrm{Li}$ by smaller $\mathrm{H}$ atoms in between layers, which increases $J_{\perp}$. Furthermore, the in-plane bond length is also larger, which reduces the scale of in-plane exchange interactions $J$. As per Eq. (29), both these factors are conducive to a larger crossover temperature $T_{*}$, where the magnetic contribution becomes large.

Other candidate materials are magnetic insulators with strong spin-orbit coupling, where the Kitaev interaction is the dominant term. Some of these materials, like $\alpha-\mathrm{RuCl}_{3}$, are believed to be proximate to a QSL phase [87]. Furthermore, the magnetic order can be suppressed by doping, making such materials an interesting playground for observing spin-liquid physics [88], although the nature of the field-induced QSL phase is still unclear.

In the layered organic insulators [6], the interplane exchange coupling is estimated to be 3 orders of magnitude below the intraplane coupling [89], and therefore, it is likely that phonons dominate the $c$-axis thermal transport at accessible temperatures. Herbertsmithite [6] is believed to have a gapped QSL ground state [46], although the spin gap seems to be quite small $\left(\Delta_{\text {gap }} \lesssim 10 \mathrm{~K}[46,85]\right)$. An applied magnetic field can induce a finite spinon density of states at zero energy, opening the way to measure the spinon contribution to $\kappa_{c}$. However, the in-plane exchange coupling $J$ is about an order of magnitude larger than in kapellasite, while the ratio $J_{\perp} / J$ is comparable in the two systems [84]. Therefore, we expect $T_{*}$ in Herbertsmithite to be smaller than in kapellasite.

Finally, we discuss a few techniques that can be used to isolate the magnetic contribution to the thermal conductivity from that of phonons.

(i) In gapless spin-liquid candidates where the magnetic contribution is a power law of the form $T^{\theta}$ with $\theta<3$, one can isolate the magnetic contribution from the phononic one (which scales as $T^{3}$ ) since the magnetic contribution is dominant at sufficiently low temperature. Plotting a curve of $\kappa / T^{\theta}$ vs $T^{3-\theta}$, the slope of the curve gives us the phonon contribution, while the intercept gives us the magnetic contribution to the thermal conductivity. This is possible as long as the sample temperature is not much higher than $T_{*}$. (ii) In addition, in some materials, an applied magnetic field may be used to establish long-range order, suppressing the spinon contribution to the thermal conductivity while weakly affecting the phonon contribution. Contrasting the measurements of the $c$-axis thermal conductivity in the presence and absence of such a field may enable us to isolate the spinon contribution.

\section{CONCLUSIONS}

We have studied the thermal conductivity in layered, gapless QSLs. The key observation is that the mechanisms of in-plane and out-of-plane thermal transport are qualitatively different: The former is carried by fractionalized excitations, while the latter is carried by gauge-neutral, nonfractionalized excitations. Thus, in all the cases we have studied, $\kappa_{a b}$ and $\kappa_{c}$ follow different power-law dependence at low temperature; in particular, the anisotropy $\kappa_{a b} / \kappa_{c}$ diverges in the limit $T \rightarrow 0$. This property is a clear hallmark of a fractionalized, layered system. A large number of layered QSL candidates have been proposed in the last few years, and interplane thermal conductivity can serve as an unambiguous probe for fractionalization in these experimental candidates.

\section{ACKNOWLEDGMENTS}

We thank S. Choi, J. Chalker, K. Michaeli, S. Kivelson, T. Senthil, S. Trebst, and M. Yamashita for useful discussions. E. B. and Y.W. were supported in part by the European Research Council (ERC) under the European Unions Horizon 2020 research and innovation program (Grant Agreement No. 639172), and by the Deutsche Forschungsgemeinschaft (CRC 183). S. M. acknowledges support from the NSF through Grant No. PHY-1656234.

\section{APPENDIX A: LAYERED KITAEV HONEYCOMB MODEL}

\section{Intralayer Hamiltonian}

We model the layered $Z_{2}$ QSL system as layers of the Kitaev honeycomb model, coupled by a weak interlayer interaction. The Kitaev honeycomb model [16] is an exactly solvable model of interacting spin- $1 / 2 \mathrm{~s}$. It is composed of a honeycomb lattice of spins interacting via direction-dependent exchange interactions,

$$
H_{0}=-J \sum_{\langle j, k\rangle} S_{j}^{\alpha_{j k}} S_{k}^{\alpha_{j k}}
$$

where $j, k$ are nearest neighbors on the hexago lattice, and $S_{j k}^{\alpha}$ are the $x, y$, or $z$ component of the spin operator, depending on the type of link between $j$ and $k$. The links are denoted $x, y$, or $z$, based on their orientation, as shown 
in Fig. 3. Each of the spins is represented in terms of Majorana fermions $b_{i}^{x}, b_{i}^{y}, b_{i}^{z}, c_{i}$ as $S_{i}^{\alpha}=i b_{i}^{\alpha} c_{i}$; however, the representation in terms of these fermions spans a larger Fock space and must be restricted to the physical Hilbert space of the spins by the gauge $D_{i}=b_{i}^{x} b_{i}^{y} b_{i}^{z} c_{i}=1$. On each $\alpha$-direction link, $u_{i j}^{\alpha}=i b_{i}^{\alpha} b_{j}^{\alpha}$ is conserved, and a theorem by Lieb [90] guarantees that the ground state is in the sector where it is possible to set $u_{i j}^{\alpha}=1$ (the flux-free sector). Thus, the ground-state manifold is described by the free Majorana Hamiltonian

$$
H_{0}=\frac{1}{2} i J \sum_{i, \delta} A_{i} B_{i+\delta}
$$

with $A_{i}$ and $B_{i+\delta}$ the $c_{i}$ Majorana on the $A$ and $B$ sublattices, respectively. The $\delta \mathrm{s}$ are the three-vectors connecting the even and odd sublattices, as shown in Fig. 3.

In order to probe the $Z_{2}$ QSL with a Fermi surface, we further consider two specific time-reversal breaking terms, which result in a Fermi surface without creating vison excitations that would take us out of the ground-state manifold:

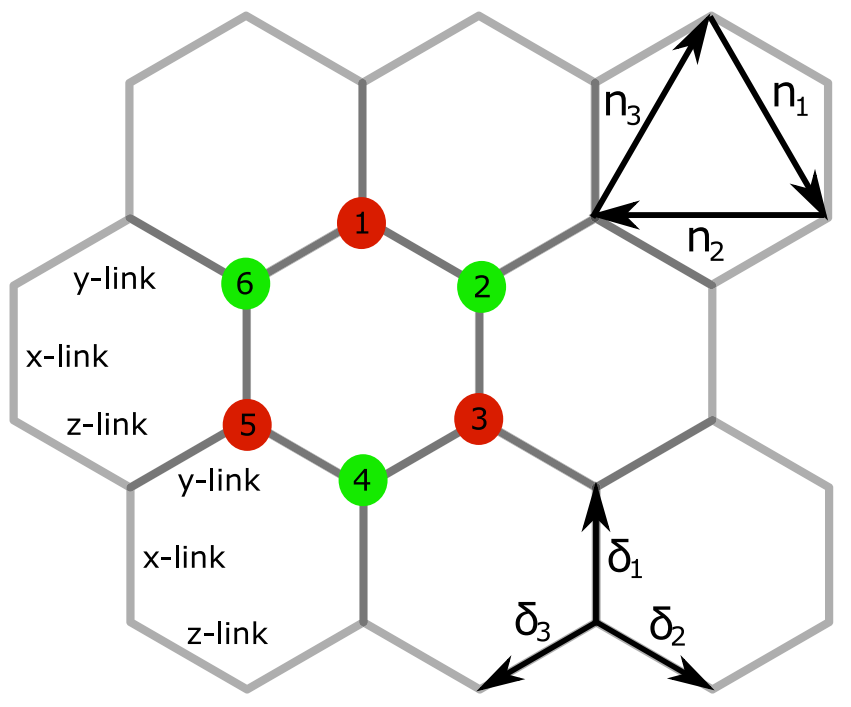

FIG. 3. The honeycomb lattice. Each unit cell is composed of an $A$ (blue) and $B$ (red) atom. Each atom in the $A$ sublattice is connected to three $B$ atoms via the vectors $\delta$, and to six $A$ atoms via the $\mathbf{n}$ vectors. The interaction between neighbors is determined by the link they share. On each plaquette, the sites are labeled as shown.

$$
\begin{aligned}
H_{\mathrm{TRB}}= & J_{\mathrm{TRB}}^{1} \sum_{\text {plaquettes }}\left[S_{1}^{z} S_{2}^{y} S_{3}^{x}-S_{2}^{x} S_{3}^{z} S_{4}^{y}+S_{3}^{y} S_{4}^{x} S_{5}^{z}-S_{4}^{z} S_{5}^{y} S_{6}^{x}+S_{5}^{x} S_{6}^{z} S_{1}^{y}-S_{6}^{y} S_{1}^{x} S_{2}^{z}\right] \\
& +J_{\text {TRB }}^{2} \sum_{\text {plaquettes }}\left[S_{1}^{z} S_{2}^{y} S_{3}^{x}+S_{2}^{x} S_{3}^{z} S_{4}^{y}+S_{3}^{y} S_{4}^{x} S_{5}^{z}+S_{4}^{z} S_{5}^{y} S_{6}^{x}+S_{5}^{x} S_{6}^{z} S_{1}^{y}+S_{6}^{y} S_{1}^{x} S_{2}^{z}\right] .
\end{aligned}
$$

Here, the sites labeled $1 \ldots 6$ on each plaquette are shown in Fig. 3.

In the ground-state manifold, these terms are given by

$$
\begin{gathered}
=-i J_{\mathrm{TRB}}^{1} \sum_{\text {plaquettes }}\left[A_{1} A_{3}-B_{2} B_{4}+A_{3} A_{5}-B_{4} B_{6}+A_{5} A_{1}-B_{6} B_{2}\right] \\
-i J_{\mathrm{TRB}}^{2} \sum_{\text {plaquettes }}\left[A_{1} A_{3}+B_{2} B_{4}+A_{3} A_{5}+B_{4} B_{6}+A_{5} A_{1}+B_{6} B_{2}\right] .
\end{gathered}
$$

We comment that on different lattices, one can also obtain a QSL with a spinon Fermi surface even in the presence of TRS [64-69].

Lastly, we also consider the effect of disorder in the system via a term $H_{\text {dis }}$, the exact form of which will be given later. Thus, the spin liquid we consider is described by

$$
H=H_{0}+H_{\mathrm{TRB}}+H_{\mathrm{dis}},
$$

with $H_{\mathrm{TRB}}=0$ in the TR-invariant case.

\section{Interlayer coupling}

The most relevant interlayer coupling terms are those that leave each layer in its ground state; that is, the interlayer coupling must commute with all the $u_{i j}^{\alpha} \mathrm{s}$. A general form of such a tunneling term in the language of the original spins will consist of spin operators from one layer coupled to spin operators from another. In order to maintain exact solvability, we consider the interlayer coupling term

$$
\begin{aligned}
H_{\perp}= & J_{\perp} \sum_{l, l^{\prime}=l \pm 1} \sum_{\text {plaquettes }}\left[S_{1, l^{z}}^{z} S_{2, l}^{y} S_{3, l}^{x}-S_{2, l^{\prime}}^{x} S_{3, l}^{z} S_{4, l}^{y}+S_{3, l}^{y} S_{4, l}^{x} S_{5, l}^{z}-S_{4, l}^{z} S_{5, l}^{y} S_{6, l}^{x}+S_{5, l}^{x} S_{6, l}^{z} S_{1, l}^{y}-S_{6, l}^{y} S_{1, l}^{x} S_{2, l}^{z}\right] \\
& \times\left[S_{1, l^{\prime}}^{z} S_{2, l^{\prime}}^{y} S_{3, l^{\prime}}^{x}-S_{2, l^{\prime}}^{x} S_{3, l^{\prime}}^{z} S_{4, l^{\prime}}^{y}+S_{3, l^{\prime}}^{y} S_{4, l^{\prime}}^{x} S_{5, l^{\prime}}^{z}-S_{4, l^{\prime}}^{z} S_{5, l^{\prime}}^{y} S_{6, l^{\prime}}^{x}+S_{5, l^{\prime}}^{x} S_{6, l^{\prime}}^{z} S_{1, l^{\prime}}^{y}-S_{6, l^{\prime}}^{y} S_{1, l^{\prime}}^{x} S_{2, l^{\prime}}^{z}\right] .
\end{aligned}
$$


In the Majorana representation, this term is given by (again setting $\hat{u}_{j, \delta}=1$ )

$$
\begin{aligned}
H_{\perp}= & J_{\perp} \sum_{l, l^{\prime}=l \pm 1} \sum_{\text {plaquettes }}\left[A_{1 l^{\prime}} A_{3, l}-B_{2, l} B_{4, l}+A_{3, l} A_{5, l}-B_{4, l} B_{6, l}+A_{5, l} A_{1, l}-B_{6, l} B_{2, l}\right] \\
& \times\left[A_{1, l^{\prime}} A_{3, l^{\prime}}-B_{2, l^{\prime}} B_{4, l^{\prime}}+A_{3, l^{\prime}} A_{5, l^{\prime}}-B_{4, l^{\prime}} B_{6, l^{\prime}}+A_{5, l^{\prime}} A_{1, l^{\prime}}-B_{6, l^{\prime}} B_{2, l^{\prime}}\right] .
\end{aligned}
$$

We have chosen this term as it allows for simple calculations, and in particular, it reduces to a "density-density" interlayer interaction [the $F_{0}$ term in Eq. (2)] in the continuum limit. We expect the exact form of the coupling term to be unimportant; the important point is that it must have at least two spinon operators from each layer. This is because only a spinon pair excitation can be transferred between different layers, and this requires at least two spinon operators from each. We comment on the case of a more generic form of the interlayer Hamiltonian, having one spin operator in each layer, in Appendix A 4 below.

\section{Continuum Hamiltonian}

Using $f_{\mathbf{k}}=\sum_{\delta} e^{i \mathbf{k} \cdot \delta}, g_{\mathbf{k}}=\sum_{i} \sin \left(\mathbf{k} \cdot \mathbf{n}_{i}\right), \psi_{l}^{A}(\mathbf{k})=e^{i \pi / 4} \sum_{j} A_{j}^{l} e^{i \mathbf{k} \cdot \mathbf{R}_{j}}, \psi_{l}^{B}(\mathbf{k})=e^{-i \pi / 4} \sum_{j} B_{j}^{l} e^{i \mathbf{k} \cdot \mathbf{R}_{j}}, \Delta_{\mathbf{k}}=4 J_{\mathrm{TRB}}^{1} g_{\mathbf{k}}$, and $m_{\mathbf{k}}=4 J_{\mathrm{TRB}}^{2} g_{\mathbf{k}}$ (where the vectors $\mathbf{n}_{i=1,2,3}$ are defined in Fig. 3), the disorder free Hamiltonian of each layer is given by

$$
\begin{aligned}
& H^{l} \equiv H_{0}^{l}+H_{\mathrm{TRB}}^{l}=\frac{1}{4} \int \frac{d^{2} k}{(2 \pi)^{2}}\left(\begin{array}{ll}
\psi_{l}^{A \dagger}(\mathbf{k}) & \psi_{l}^{B \dagger}(\mathbf{k})
\end{array}\right)\left(\begin{array}{cc}
\Delta_{\mathbf{k}}+m_{\mathbf{k}} & J f_{\mathbf{k}} \\
J f_{\mathbf{k}}^{*} & \Delta_{\mathbf{k}}-m_{\mathbf{k}}
\end{array}\right)\left(\begin{array}{c}
\psi_{l}^{A}(\mathbf{k}) \\
\psi_{l}^{B}(\mathbf{k})
\end{array}\right) \\
& H_{\perp}=\frac{1}{2} J_{\perp} \sum_{l, l^{\prime}=l \pm 1} \int \frac{d^{2} k}{(2 \pi)^{2}} \frac{d^{2} k^{\prime}}{(2 \pi)^{2}} \frac{d^{2} q}{(2 \pi)^{2}} g_{\mathbf{k}+\mathbf{k}^{\prime}}\left[\psi_{l}^{A \dagger}(\mathbf{k}) \psi_{l}^{A}(\mathbf{k}+\mathbf{q})+\psi_{l}^{B^{\dagger}}(\mathbf{k}) \psi_{l}^{B}(\mathbf{k}+\mathbf{q})\right] \\
& \times\left[\psi_{l^{\prime}}^{A \dagger}\left(\mathbf{k}^{\prime}\right) \psi_{l^{\prime}}^{A}\left(\mathbf{k}^{\prime}-\mathbf{q}\right)+\psi_{l^{\prime}}^{B^{\dagger}}\left(\mathbf{k}^{\prime}\right) \psi_{l^{\prime}}^{B}\left(\mathbf{k}^{\prime}-\mathbf{q}\right)\right] .
\end{aligned}
$$

The low-energy theory is centered near the Dirac points $\mathbf{K}, \quad \mathbf{K}^{\prime}=[(2 \pi) / 3][ \pm(1 / \sqrt{3}), 1]$. Near these points, $J f_{\mathbf{k}} \approx v\left(k x \pm i k_{y}\right)$, with $v=3 J / 2$. The low-energy inplane Hamiltonian can thus be written as two Majorana theories with a Dirac dispersion. It is convenient to consider an equivalent system, of complex fermions that reside only on half the Brillouin zone; this makes use of the equivalence $\psi^{A}(\mathbf{k})=\psi^{A \dagger}(-\mathbf{k})$. The low-energy theory of this system is given by a single Dirac cone centered at $\mathbf{K}$, and its in-plane Hamiltonian is given by $H^{l}=\int\left[\left(d^{2} k\right) /\right.$ $\left.(2 \pi)^{2}\right] \psi_{\mathbf{k}}^{l \dagger} H(\mathbf{k}) \psi_{\mathbf{k}}^{l}$, with

$$
H(\mathbf{k})=v \boldsymbol{\sigma} \cdot \mathbf{k}+m \sigma_{z}+\Delta
$$

Here, $\psi_{\mathbf{k}}^{\dagger l}=\left(\psi_{l}^{A \dagger}(\mathbf{k}), \psi_{l}^{B^{\dagger}}(\mathbf{k})\right)$ is a spinor of complex fermions, $\boldsymbol{\sigma}=\left(\sigma_{x}, \sigma_{y}\right)$ a vector of Pauli matrices, $\Delta=\Delta_{\mathbf{k}=\mathbf{K}}$, and $m=m_{\mathbf{k}=\mathbf{K}}$. This result is Eq. (1) in the main text.

In terms of the continuum theory, the interplane term in Eq. (A7) is given by (neglecting the variation of $\Delta_{\mathbf{k}}$ around the Dirac points)

$$
\begin{aligned}
H_{\perp}= & \frac{1}{2} J_{\perp} \sum_{l, l^{\prime}=l \pm 1} \int \frac{d^{2} k}{(2 \pi)^{2}} \frac{d^{2} k^{\prime}}{(2 \pi)^{2}} \frac{d^{2} q}{(2 \pi)^{2}} \\
& \times\left\{g_{\mathbf{k}+\mathbf{k}^{\prime}}\left[\psi_{l}^{A \dagger}(\mathbf{k}) \psi_{l}^{A}(\mathbf{k}+\mathbf{q})+\psi_{l}^{B^{\dagger}}(\mathbf{k}) \psi_{l}^{B}(\mathbf{k}+\mathbf{q})\right]\left[\psi_{l^{\prime}}^{A \dagger}\left(\mathbf{k}^{\prime}\right) \psi_{l^{\prime}}^{A}\left(\mathbf{k}^{\prime}-\mathbf{q}\right)+\psi_{l^{\prime}}^{B^{\dagger}}\left(\mathbf{k}^{\prime}\right) \psi_{l^{\prime}}^{B}\left(\mathbf{k}^{\prime}-\mathbf{q}\right)\right]\right. \\
& \left.+g_{\mathbf{k}-\mathbf{k}^{\prime}}\left[\psi_{l}^{A}(-\mathbf{k}) \psi_{l}^{A}(\mathbf{k}+\mathbf{q})+\psi_{l}^{B}(-\mathbf{k}) \psi_{l}^{B}(\mathbf{k}+q)\right]\left[\psi_{l^{\prime}}^{A \dagger}\left(\mathbf{k}^{\prime}\right) \psi_{l^{\prime}}^{A \dagger}\left(-\mathbf{k}^{\prime}+\mathbf{q}\right)+\psi_{l^{\prime}}^{B^{\dagger}}\left(\mathbf{k}^{\prime}\right) \psi_{l^{\prime}}^{B^{\dagger}}\left(-\mathbf{k}^{\prime}+\mathbf{q}\right)\right]+\text { H.c. }\right\} .
\end{aligned}
$$

We expand the $g_{\mathbf{k}}$ form factors for small deviations away from the Dirac point $\mathbf{K}$; we set $F_{0}=g_{2 \mathbf{K}}$, while the lowestorder term in the expansion of $g_{\mathbf{k}-\mathbf{k}^{\prime}}$ away from the $\mathbf{K}$ point vanishes, introducing additional factors of momentum. This method will introduce additional factors of temperature $T$ in the contribution to the thermal conductivity, and we therefore neglect the pair hopping term.
We work in the basis of the eigenstates of $H_{0}^{l}+H_{\mathrm{TRB}}^{l}$. In the TR symmetric case, where $\Delta=m=0$, the eigenstates are given by $a_{\lambda}(\mathbf{k})=\left[\psi^{A}(\mathbf{k})+e^{i \phi_{\mathbf{k}}} \psi^{B}(\mathbf{k})\right] / \sqrt{2}$, where $\phi_{\mathbf{k}}$ is the angle between $k_{x}$ and $k_{y}$, and their energies are $\epsilon_{\mathbf{k}}^{\lambda}=\lambda v k$. For simplicity, in the analysis of the $Z_{2}$ QSL with a Fermi surface, we consider the regime $\Delta>m>0$, $m \gg \Delta-m$. In this limit, the eigenstates with energy 
close to the Fermi surface are located almost entirely on the $A$ sublattice, and we may ignore the sublattice degree of freedom; these eigenstates are denoted by $a(\mathbf{k}) \sim$ $\psi^{A}(\mathbf{k})$. In this basis, the single-layer Green's functions are $G\left(\mathbf{k}, i \nu_{n}\right)=\left[i \nu_{n}-k^{2} / 2 m^{*}+\mu\right]^{-1}$, with $\mu=\Delta-m$ and $m^{*}=v^{2} / m$.

\section{Generic interlayer Hamiltonian}

The interlayer coupling term (A6) is designed to maintain the exact solvability of the model and for computational convenience. However, generically, we expect the largest components of the interlayer coupling Hamiltonian to be quadratic in the spin operators. Within the Kitaev model, a quadratic interlayer coupling term (such as a Heisenberg term, $J_{\perp}^{\prime} \sum_{\alpha} S_{l}^{\alpha} S_{l+1}^{\alpha}$, with $\alpha=x, y, z$ ) does not merely create a pair of spinon excitations in each layer. Rather, it creates a pair of spinons and a pair of gapped vison (flux) excitations. In order to annihilate the pair of flux excitations and return to the low-energy subspace, we have to apply the $J_{\perp}^{\prime}$ term again. Thus, it appears that the effective interplane interaction in the low-energy effective Hamiltonian (2) is proportional to $J_{\perp} \sim\left(J_{\perp}^{\prime}\right)^{2} / \Delta_{v}$, where $J_{\perp}^{\prime}$ is the "microscopic" strength of the interlayer coupling and $\Delta_{v}$ is the vison gap. One may then worry that the effective interplane interaction $J_{\perp}$ is too small to contribute significantly to the interlayer thermal conductivity.

However, we argue that for a generic intralayer Hamiltonian that also contains non-Kitaev terms (such that even the intralayer Hamiltonian is not exactly solvable), this is not the case; in fact, $J_{\perp} \propto J_{\perp}^{\prime}$ because, in the generic case, the vison excitations are not static, even within the single-layer Hamiltonian. A pair of vison excitations can annihilate each other without the need for another application of the interlayer $J_{\perp}^{\prime}$ term.

To illustrate this idea, consider the case where there is an additional intraplane interaction:

$$
H_{\Gamma}=\sum_{\langle i, j\rangle, l, \alpha, \beta} \Gamma_{\alpha \beta} S_{i, l}^{\alpha} S_{j, l}^{\beta},
$$

where $\langle i, j\rangle$ denotes two nearest-neighbor sites $i, j$ on the honeycomb lattice, and $\Gamma_{\alpha \beta}$ is a $3 \times 3$ symmetric matrix. Such terms are present in real "Kitaev materials" [91].

Consider a quadratic interplane coupling term of the form $J_{\perp}^{\prime} S_{1, l}^{z} S_{1, l+1}^{z}$, where the position of site 1 is indicated in Fig. 3. We can now derive the effective interplane coupling $J_{\perp}$ (that creates a pair of spinons in each layer and does not create any visons) perturbatively in both $J_{\perp}^{\prime}$ and $\Gamma_{\alpha \beta}$. One can check explicitly that using the following sequence of operators,

$$
S_{1, l}^{z}\left(S_{1, l}^{x} S_{2, l}^{x}\right)\left(S_{1, l}^{y} S_{2, l}^{x}\right) \times(l \rightarrow l+1),
$$

amounts, in a certain gauge, to using $c_{1, l} c_{2, l} c_{1, l+1} c_{2, l+1}$ and not changing the number of visons in either layer. The strength of this term is $J_{\perp} \sim\left\{\left[J_{\perp}^{\prime}\left(\Gamma_{x x} \Gamma_{x y}\right)^{2}\right] / \Delta_{v}^{4}\right\}$. Thus, the term in the effective Hamiltonian that creates a pair of fermionic spinons in each of the adjacent layers $l, l+1$ is proportional to $J_{\perp}^{\prime}$. Generically, there is no reason to expect $\Gamma_{\alpha \beta}$ to be much smaller in magnitude than $\Delta_{v}$ since both energy scales characterize the intraplane Hamiltonian and do not involve interlayer coupling. We conclude that in a generic situation, $J_{\perp}$ and $J_{\perp}^{\prime}$ are of the same order of magnitude.

\section{APPENDIX B: EVALUATION OF THE INTEGRAL IN EQ. (4)}

The calculation proceeds similarly to the computation of the lifetime of a quasiparticle in a Fermi liquid. We integrate over $\mathbf{k}, \mathbf{k}^{\prime}$ first, fixing $\mathbf{q}$. Let us choose the axes such that $\mathbf{q}$ is in the $x$ direction. For $q<2 k_{F}$, there are pairs of points on the Fermi surface that are connected by $\mathbf{q}$; we denote these points by $\mathbf{k}_{0}, \mathbf{k}_{0}+\mathbf{q}$. Then, we parametrize

$$
\begin{aligned}
& \mathbf{k}=\mathbf{k}_{0}+\delta \mathbf{k}, \\
& \mathbf{k}=-\mathbf{k}_{0}+\delta \mathbf{k}^{\prime} .
\end{aligned}
$$

It is convenient to linearize the dispersion around the Fermi surface; then, to leading order in $\delta k$,

$$
\begin{aligned}
\epsilon_{\mathbf{k}} & =v \delta k_{x} \sin \theta+v \delta k_{y} \cos \theta, \\
\epsilon_{\mathbf{k}+\mathbf{q}} & =-v \delta k_{x} \sin \theta+v \delta k_{y} \cos \theta, \\
\epsilon_{\mathbf{k}^{\prime}} & =-v \delta k_{x} \sin \theta-v \delta k_{y} \cos \theta, \\
\epsilon_{\mathbf{k}^{\prime}-\mathbf{q}} & =v \delta k_{x} \sin \theta-v \delta k_{y} \cos \theta .
\end{aligned}
$$

Here, $\sin \theta=\left[q /\left(2 k_{F}\right)\right]$ (see Fig. 4), and $v=\left[\left(k_{F}\right) / m^{*}\right]$ is the Fermi velocity. The integrals over $\delta \mathbf{k}, \delta \mathbf{k}^{\prime}$ can now be performed easily, giving

$$
\begin{aligned}
\kappa_{c}= & 2 \pi \frac{J_{\perp}^{2}}{T^{2}} \int d \epsilon_{1} d \epsilon_{2} d \epsilon_{3}\left(1-n_{F}\left(\epsilon_{1}\right)\right) n_{F}\left(\epsilon_{2}\right) \\
& \times\left(1-n_{F}\left(\epsilon_{3}\right)\right) n_{F}\left(\epsilon_{1}-\epsilon_{2}+\epsilon_{3}\right) \times\left(\epsilon_{1}-\epsilon_{2}\right)^{2} \\
& \times \int \frac{d^{2} q}{(2 \pi)^{6}} \frac{1}{v_{F}^{4} \cos ^{2} \theta \sin ^{2} \theta} .
\end{aligned}
$$

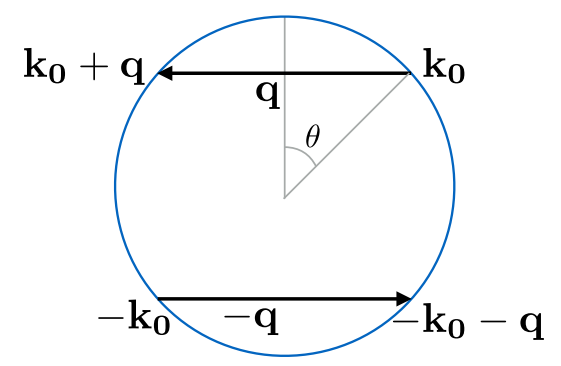

FIG. 4. Choice of $\mathbf{k}_{0}$ for a given $\mathbf{q}$ when integrating over $\mathbf{k}, \mathbf{k}^{\prime}$ in Eq. (4). The blue circle is the Fermi surface. 
The integral over $\epsilon_{1,2,3}$ can now be performed; by scaling, this integral is proportional to $T^{5}$. Substituting $\theta$ with $q$, we get

$$
\kappa_{c} \propto \frac{J_{\perp}^{2}}{v_{F}^{4}} T^{3} \int_{0}^{2 k_{F}} d q \frac{k_{F}^{2}}{q\left[1-q^{2} /\left(4 k_{F}^{2}\right)\right]} .
$$

Using $\nu=\left[k_{F} /\left(2 \pi v_{F}\right)\right]$, we arrive at Eq. (4) in the text. The logarithmic divergence comes from small $\mathbf{q}$ scattering, as well as from scattering with momentum transfer close to $q=2 k_{F}$. The inelastic lifetime of the quasiparticles in each layer, $\tau \propto 1 / T^{2}$, is needed in order to cut off this divergence. There is another contribution from $q>2 k_{F}$; this contribution is parametrically the same as Eq. (B4).

\section{APPENDIX C: DISORDERED $Z_{2}$ QSL}

\section{Possible forms of disorder}

In a TR-invariant system, we consider disorder in the spin-spin couplings $J$ :

$$
H_{\mathrm{dis}}=\sum_{\langle j, k\rangle} \delta J_{j k} S_{j}^{\alpha_{j k}} S_{k}^{\alpha_{j k}},
$$

where, again, $j, k$ are nearest neighbors and $\alpha_{j k}=x, y, z$, according to the type of link. In the Majorana fermion representation, this becomes

$$
\begin{aligned}
H_{\text {dis }} & =-\frac{1}{2} i \sum_{i} \delta J_{i, i+\delta} A_{i} B_{i+\delta} \\
& =v \int \frac{d^{2} k d^{2} k^{\prime}}{(2 \pi)^{4}} \psi_{\mathbf{k}}^{\dagger} \mathcal{A}_{\mathbf{k}, \mathbf{k}^{\prime}} \cdot \sigma \psi_{\mathbf{k}^{\prime}},
\end{aligned}
$$

with $\mathcal{A}_{\mathbf{k}, \mathbf{k}^{\prime}}{ }^{x}=(1 / J) \sum_{j, \delta} \operatorname{Re}\left[e^{-i k^{\prime} \delta} \delta J_{j, \delta} e^{i\left(\mathbf{k}-\mathbf{k}^{\prime}\right) R_{j}}\right], \mathcal{A}_{\mathbf{k}, \mathbf{k}^{\prime}}{ }^{y}=$ $(1 / J) \sum_{j, \delta} \Im\left[e^{-i k^{\prime} \delta} \delta J_{j, \delta} e^{i\left(\mathbf{k}-\mathbf{k}^{\prime}\right) R_{j}}\right]$; thus, the low-energy, long-wavelength disorder is of the form of a random vector potential.

Disorder that affects the next-nearest-neighbor hopping results from three-spin interaction terms in the original spin model; these terms break time-reversal symmetry. Depending on the relative sign of the disorder between the $A$ and $B$ sublattices, just as in Eq. (A4), these terms will give rise to a scalar potential term

$$
H_{\mathrm{dis}}=\int \frac{d^{2} k d^{2} k^{\prime}}{(2 \pi)^{4}} \mathcal{V}_{\mathbf{k}, \mathbf{k}^{\prime}} \psi_{\mathbf{k}}^{\dagger} \psi_{\mathbf{k}^{\prime}},
$$

or a random mass term

$$
H_{\mathrm{dis}}=\int \frac{d^{2} k d^{2} k^{\prime}}{(2 \pi)^{4}} \psi_{\mathbf{k}}^{\dagger} \mathcal{M}_{\mathbf{k}, \mathbf{k}^{\prime}} \sigma^{z} \psi_{\mathbf{k}^{\prime}}
$$

\section{Interlayer thermal conductivity}

To compute the $c$-axis conductivity in a disordered, layered $Z_{2}$ QSL, we compute the rate at which pairs of spinon excitations tunnel between planes. This computation can be done using the Fermi golden rule, analogously to Eq. (4), replacing the momentum eigenstates with eigenstates of the disordered intraplane Hamiltonian.

The general expression for the thermal conductivity is

$$
\begin{aligned}
\kappa_{c}= & \frac{2 \pi J_{\perp}^{2}}{Z T^{2}} \sum_{i, f} \sum_{l, l^{\prime}} e^{-\left(E_{i, l} / T\right)-\left(E_{i, l^{\prime}} / T\right)}\left(E_{i, l}-E_{f, l}\right)^{2} \\
& \times\left|\left\langle f\left|H_{\perp}\left(l, l^{\prime}\right)\right| i\right\rangle\right|^{2} \times \delta\left(E_{i, l}+E_{i, l^{\prime}}-E_{f, l}-E_{f, l}\right) .
\end{aligned}
$$

Here, $H_{\perp}\left(l, l^{\prime}\right)$ is the part of the interplane Hamiltonian that couples layers $l$ and $l^{\prime}$, and $|i, f\rangle$ are the initial and final many-body eigenstates of the system with $J_{\perp}=0$ (decoupled planes), with corresponding energies $E_{i, l}$ and $E_{f, l}$ (at layer $l$ ). Since we neglect intraplane interactions, we can expand the fermionic spinon operators in the basis of the single-particle eigenstates of each layer (which include the effects of disorder):

$$
\psi_{l}^{\eta}(x)=\sum_{\lambda} \varphi_{l, \lambda, \eta}(x) f_{\lambda, l}
$$

where $\eta=A, B$ labels the sublattice, and $f_{\lambda, l}$ annihilates an eigenstate with energy $\xi_{\lambda, l}$, which has the wave function $\varphi_{l, \lambda, \eta}(x)$.

As in the main text, we mostly work with an interlayer Hamiltonian of the "density-density" form, $H_{\perp}\left(l, l^{\prime}\right)=$ $\sum_{\eta, \eta^{\prime}} \int d^{2} x \psi_{l}^{\eta^{\dagger}}(x) \psi_{l}^{\eta}(x) \psi_{l^{\prime}}^{\eta^{\dagger}}(x) \psi_{l^{\prime}}^{\eta}(x)$, commenting along the way about other forms of $H_{\perp}$. Using Eq. (C6), we can write the disorder-averaged $c$-axis thermal conductivity as

$$
\begin{aligned}
\kappa_{c}= & \frac{2 \pi J_{\perp}^{2}}{T^{2}} \sum_{\lambda_{1} \ldots 4} \sum_{\eta_{11 . \ldots 4}} n_{F}\left(\xi_{l \lambda_{1}}\right)\left[1-n_{F}\left(\xi_{l \lambda_{3}}\right)\right] n_{F}\left(\xi_{l^{\prime} \lambda_{2}}\right)\left[1-n_{F}\left(\xi_{l^{\prime} \lambda_{4}}\right)\right]\left(\xi_{l \lambda_{1}}-\xi_{l \lambda_{3}}\right)^{2} \delta\left(\xi_{l \lambda_{1}}+\xi_{l^{\prime} \lambda_{2}}-\xi_{l \lambda_{3}}-\xi_{l^{\prime} \lambda_{4}}\right) \\
& \times \int d^{2} x d^{2} x^{\prime}\left\langle\varphi_{l \lambda_{1} \eta_{1}}^{*}(x) \varphi_{l \lambda_{3} \eta_{1}}(x) \varphi_{l \lambda_{1} \eta_{2}}\left(x^{\prime}\right) \varphi_{l \lambda_{3} \eta_{2}}^{*}\left(x^{\prime}\right) \varphi_{l^{\prime} \lambda_{2} \eta_{3}}^{*}(x) \varphi_{l^{\prime} \lambda_{4} \eta_{3}}(x) \varphi_{l^{\prime} \lambda_{2} \eta_{4}}\left(x^{\prime}\right) \varphi_{l^{\prime} \lambda_{4} \eta_{4}}^{*}\left(x^{\prime}\right)\right\rangle_{\mathrm{dis}},
\end{aligned}
$$

where $\langle\ldots\rangle_{\text {dis }}$ represents disorder averaging. 
We denote

$$
g_{l}\left(q, \varepsilon, \varepsilon^{\prime}\right)=\sum_{\lambda_{1,2}, \eta, \eta^{\prime}} \int d^{2} x e^{-i q \cdot x}\left\langle\varphi_{l \lambda_{1} \eta}^{*}(x) \varphi_{l \lambda_{2} \eta}(x) \varphi_{l \lambda_{1} \eta^{\prime}}(0) \varphi_{l \lambda_{2} \eta^{\prime}}^{*}(0)\right\rangle_{\mathrm{dis}} \delta\left(\varepsilon-\xi_{l, \lambda_{1}}\right) \delta\left(\varepsilon^{\prime}-\xi_{l, \lambda_{2}}\right),
$$

such that the thermal conductivity is given by

$$
\begin{aligned}
\kappa_{c}= & \frac{2 \pi J_{\perp}^{2}}{T^{2}} \int d \epsilon_{1} d \epsilon_{2} d \omega n_{F}\left(\epsilon_{1}\right)\left[1-n_{F}\left(\epsilon_{1}+\omega\right)\right] n_{F}\left(\epsilon_{2}\right)\left[1-n_{F}\left(\epsilon_{2}-\omega\right)\right] \omega^{2} \\
& \times \int d^{2} q g_{l}\left(q, \epsilon_{1}, \epsilon_{1}+\omega\right) g_{l}\left(-q, \epsilon_{2}, \epsilon_{2}-\omega\right) .
\end{aligned}
$$

The remaining task is to compute the function $g_{l}\left(\mathbf{q}, \varepsilon, \varepsilon^{\prime}\right)$ for a $Z_{2}$ QSL with either a Dirac spectrum or a Fermi surface.

\section{a. Disordered Dirac}

The properties of two-dimensional Dirac fermions coupled to a random vector potential, which corresponds to a disordered $Z_{2}$ Dirac QSL with time-reversal symmetry, has been studied extensively in Ref. [73]. Here, we briefly review some of the results of Ref. [73] and use them to determine the scaling of the $c$-axis thermal conductivity with temperature.

Since the problem is noninteracting, the actions for different frequency modes decouple (before disorder averaging). The $\omega=0$ system is described by a fixed line of interacting theories in $d=1+1$ dimensions [73]. The frequency $\omega$ corresponds to a relevant operator with scaling dimension $2-z$, where $z=1+\Delta_{A} / \pi$ is the dynamical critical exponent, and $\Delta_{A}$ is the disorder strength, defined in Eq. (11). In other words, under scaling, $q \rightarrow q^{\prime}=q / b$, $\omega \rightarrow \omega^{\prime}=\omega / b^{z}$. The fixed line is characterized by $\omega / T$ scaling.

The function $g_{l}\left(q, \varepsilon, \varepsilon^{\prime}\right)$ satisfies the scaling relation

$$
g_{l}\left(q, \varepsilon_{1}, \varepsilon_{2}\right)=b^{-y} g_{l}\left(b q, b^{z} \varepsilon_{1}, b^{z} \varepsilon_{2}\right),
$$

where $y$ is a critical exponent related to the scaling dimension of the fermion density operator, which we compute below, and $b$ is a rescaling factor. Choosing $b=\left|\varepsilon_{1}\right|^{-1 / z}$, we find that $g_{l}$ can be written as

$$
g_{l}\left(q, \varepsilon_{1}, \varepsilon_{2}\right)=\left|\varepsilon_{1}\right|^{y / z} \Phi\left(\frac{q}{\left|\varepsilon_{1}\right|^{1 / z}}, \frac{\varepsilon_{2}}{\left|\varepsilon_{1}\right|}\right),
$$

where $\Phi$ is a universal scaling function.

To determine $y$, we notice that $g_{l}$ is related to the densitydensity correlator:

$$
\begin{aligned}
\chi\left(q, \omega_{n}\right) & \equiv\left\langle n\left(q, \omega_{n}\right) n\left(-q, \omega_{n}\right)\right\rangle \\
& =\int d^{2} x e^{-i q \cdot x} \sum_{\eta, \eta^{\prime}} \sum_{\alpha, \gamma} \frac{n_{F}\left(\varepsilon_{\alpha}\right)-n_{F}\left(\varepsilon_{\gamma}\right)}{i \omega_{n}-\varepsilon_{\alpha}+\varepsilon_{\gamma}}\left\langle\varphi_{l \alpha \eta}^{*}(x) \varphi_{l \gamma \eta}(x) \varphi_{l \alpha \eta^{\prime}}(0) \varphi_{l \gamma \eta^{\prime}}^{*}(0)\right\rangle_{\mathrm{dis}} \\
& =\int d \varepsilon d \varepsilon^{\prime} \frac{n_{F}(\varepsilon)-n_{F}\left(\varepsilon^{\prime}\right)}{i \omega_{n}-\varepsilon+\varepsilon^{\prime}} g_{l}\left(q, \varepsilon, \varepsilon^{\prime}\right),
\end{aligned}
$$

where $n_{F}(\varepsilon)$ is the Fermi function. Using Eq. (C11), this can be written as

$$
\chi\left(q, \omega_{n}\right)=T^{1+y / z} \int d \xi d \xi^{\prime} \frac{n_{F}(T \xi)-n_{F}\left(T \xi^{\prime}\right)}{i \omega_{n}-\xi+\xi^{\prime}}|\xi|^{y / z} \Phi\left(\frac{q}{T|\xi|^{1 / z}}, \frac{\xi^{\prime}}{|\xi|}\right) .
$$

Here, we have used Eq. (C11) and performed a change of variables, $\varepsilon=T \xi, \varepsilon^{\prime}=T \xi^{\prime}$. On the other hand, $\chi\left(q, \omega_{n}=0\right)$ can be expressed as

$$
\begin{aligned}
\chi\left(q, \omega_{n}=0, T\right) & =\int_{0}^{\beta} d \tau \int d^{2} x e^{-i q \cdot x}\left\langle n_{l}(x, \tau) n_{l}(0,0)\right\rangle=T \sum_{\nu, \nu^{\prime}} \int d^{2} x e^{-i q \cdot x}\left\langle n_{l, \nu}(x) n_{l, \nu^{\prime}}(0)\right\rangle \\
& =T \sum_{\nu, \nu^{\prime}} \int d^{2} x e^{-i q \cdot x} b^{-2(2-z)}\left\langle n_{l, \nu}(x / b) n_{l, \nu^{\prime}}(0)\right\rangle=b^{2(z-1)} T \chi\left(b q, \omega=0, b^{z} T\right) .
\end{aligned}
$$


Here, $n_{l, \nu}(x)$ is the Matsubara frequency $\nu$ component of the density operator in layer $l$ [92]. In the second to last line, we have applied scaling to the correlation function $\left\langle n_{l, \nu}(x) n_{l, \nu^{\prime}}(0)\right\rangle$, using the fact that the scaling dimension of $n_{l, \nu}(x)$ is $2-z$ [73].

Choosing $b=T^{-1 / z}$ in Eq. (C14), we get $\chi\left(q, \omega_{n}=0, T\right)=T^{[(2-z) / z]} \Psi\left(q / T^{1 / z}\right)$, where $\Psi$ is a scaling function. Comparing this to Eq. (C12), we can extract the exponent $y$ :

$$
y=2(1-z)
$$

Now, we are in a position to find the scaling of the $c$-axis thermal conductivity with temperature. Inserting Eq. (C11) into Eq. (C9) results in

$$
\begin{aligned}
\kappa_{c}= & \frac{2 \pi J_{\perp}^{2}}{T^{2}} \int d \varepsilon_{1} d \varepsilon_{2} d \omega n\left(\varepsilon_{1}\right)\left[1-n\left(\varepsilon_{1}+\omega\right)\right] n\left(\varepsilon_{2}\right) \\
& \times\left[1-n\left(\varepsilon_{2}-\omega\right)\right] \omega^{2} \int d^{2} q\left|\varepsilon_{1}\right|^{y / z} \Phi \\
& \times\left(\frac{q}{\left|\varepsilon_{1}\right|^{1 / z}}, \frac{\varepsilon_{1}-\omega}{\left|\varepsilon_{1}\right|}\right)\left|\varepsilon_{2}\right|^{y / z} \Phi\left(-\frac{q}{\left|\varepsilon_{2}\right|^{1 / z}}, \frac{\varepsilon_{2}-\omega}{\left|\varepsilon_{2}\right|}\right)
\end{aligned}
$$

Rescaling the integral, $\tilde{\omega}=\omega / T, \tilde{\varepsilon}_{1,2}=\varepsilon_{1,2} / T$, and $\tilde{q}=q / T^{1 / z}$, we get

$\kappa_{c} \sim \frac{2 \pi J_{\perp}^{2}}{T^{2}} T^{2 / z}\left(T^{3}\right)\left(T^{2}\right)\left(T^{4(1-z) / z}\right) \sim J_{\perp}^{2} T^{(6-z) / z}$.

This result coincides with that of the clean case in the limit $\Delta_{A} \rightarrow 0$ (i.e., $z=1+\Delta_{A} / \pi \rightarrow 1$ ).

The analysis above has been done for an interplane interaction of the density-density form. A similar analysis can be done for any quartic interplane interaction. The only difference is the scaling dimension of the fermion bilinear operator that appears in the interaction term, which can be determined using the methods of Ref. [73]. It turns out, however, that for any $\Delta_{A}>0$, the density operator is the fermion bilinear with the smallest scaling dimension. Hence, a density-density interaction gives the dominant contribution to $\kappa_{c}$ at low temperatures.

\section{b. Disordered FS}

In the disordered FS case, we know that the densitydensity correlation function takes a diffusive form at small $q, \omega_{n}$ :

$$
\chi\left(q, \omega_{n}\right)=\nu \frac{D q^{2}}{\left|\omega_{n}\right|+D q^{2}},
$$

where $D$ is the diffusion constant, and $\nu$ is the density of states at the Fermi level. Comparing this result to Eq. (C12), we deduce that $g_{l}\left(q, \varepsilon, \varepsilon^{\prime}\right)$ should satisfy the following scaling relation:

$$
g_{l}\left(q, \varepsilon, \varepsilon^{\prime}\right)=b^{2} g_{l}\left(b q, b^{2} \varepsilon, b^{2} \varepsilon^{\prime}\right) .
$$

Hence, $g_{l}\left(q, \varepsilon, \varepsilon^{\prime}\right)$ can be written as

$$
g_{l}\left(q, \varepsilon, \varepsilon^{\prime}\right)=\frac{\nu}{D q^{2}} \Omega\left(\frac{\varepsilon}{D q^{2}}, \frac{\varepsilon^{\prime}}{D q^{2}}\right)
$$

where $\Omega\left(\xi, \xi^{\prime}\right)$ is a dimensionless scaling function.

We may now use this form in Eq. (C9) to get

$$
\begin{aligned}
\kappa_{c}= & \frac{2 \pi J_{\perp}^{2}}{T^{2}} \int d \varepsilon_{1} d \varepsilon_{2} d \omega n\left(\varepsilon_{1}\right)\left[1-n\left(\varepsilon_{1}+\omega\right)\right] n\left(\varepsilon_{2}\right) \\
& \times\left[1-n\left(\varepsilon_{2}-\omega\right)\right] \omega^{2} \int d^{2} q \frac{\nu^{2}}{D^{2} q^{4}} \Omega\left(\frac{\varepsilon_{1}}{D q^{2}}, \frac{\varepsilon_{1}-\omega}{D q^{2}}\right) \\
& \times \Omega\left(\frac{\varepsilon_{2}}{D q^{2}}, \frac{\varepsilon_{2}-\omega}{D q^{2}}\right) .
\end{aligned}
$$

Changing variables to $\tilde{\varepsilon}_{1,2}=\varepsilon_{1,2} / T, \quad \tilde{q}=q / \sqrt{D T}$, we obtain

$$
\kappa_{c} \sim J_{\perp}^{2} \nu^{2} \frac{T^{2}}{D} .
$$

This result coincides with the result of the Kubo formula calculation described in the main text.

\section{APPENDIX D: KUBO FORMULA FOR THERMAL CONDUCTIVITY}

\section{Thermal current operator}

The systems we consider consist of layers of quasi-2D QSLs, described by the in-plane Hamiltonian $H^{l}$ and coupled by interplane hopping terms, which may be written as sums of terms of the form $H_{\perp}=J_{\perp} O^{l} O^{l+1}$, where $O^{l}$ is composed of operators of the $l$ level only. The energy density of a single layer $l$ is thus (for a single term $H_{\perp}$; the extension to a sum of terms is straightforward)

$$
E^{l}=H^{l}+\frac{1}{2} J_{\perp}\left\{O^{l} O^{l-1}+O^{l} O^{l+1}\right\} .
$$

Its time derivative is then 


$$
\begin{aligned}
\dot{E}^{l}= & i J_{\perp}\left\{\left[O^{l}, H^{l}\right] O^{l-1}+\left[O^{l}, H^{l}\right] O^{l+1}+\frac{1}{2}\left[H^{l}, O^{l}\right] O^{l-1}\right. \\
& +\frac{1}{2}\left[H^{l}, O^{l}\right] O^{l+1}+\frac{1}{2}\left[H^{l-1}, O^{l-1}\right] O^{l} \\
& \left.+\frac{1}{2}\left[H^{l+1}, O^{l+1}\right] O^{l}\right\}+O\left(J_{\perp}^{2}\right) \\
= & \frac{1}{2} i J_{\perp}\left\{\left[H^{l-1}, O^{l-1}\right] O^{l}-\left[H^{l}, O^{l}\right] O^{l-1}\right. \\
& \left.+\left[H^{l+1}, O^{l+1}\right] O^{l}-\left[H^{l}, O^{l}\right] O^{l+1}\right\}+O\left(J_{\perp}^{2}\right) \\
= & \frac{1}{2} J_{\perp}\left\{\dot{O}^{l-1} O^{l}-\dot{O}^{l} O^{l-1}+\dot{O}^{l+1} O^{l}-\dot{O}^{l} O^{l+1}\right\} \\
& +O\left(J_{\perp}^{2}\right),
\end{aligned}
$$

where in the last equality, we use the fact that to lowest order in $J_{\perp}, \dot{O}^{l}=i\left[H^{l}, O^{l}\right]$.

The thermal current operator is given by [93]

$$
J^{Q}=\sum_{l} l \dot{E}^{l}
$$

and therefore,

$$
\begin{aligned}
J^{Q}= & \frac{1}{2} J_{\perp} \sum_{l}\left\{(l+1) \dot{O}^{l} O^{l+1}-l \dot{O}^{l} O^{l-1}\right. \\
& \left.+(l-1) \dot{O}^{l} O^{l-1}-l \dot{O}^{l} O^{l+1}\right\}+O\left(J_{\perp}^{2}\right) \\
= & \sum_{l} J_{l, l+1}^{Q}+O\left(J_{\perp}^{2}\right)
\end{aligned}
$$

with $J_{l, l+1}^{Q}=\frac{1}{2} J_{\perp}\left(\dot{O}^{l} O^{l+1}-O^{l} \dot{O}^{l+1}\right)$. This formula satisfies the continuity equation $J_{l, l+1}^{Q}-J_{l-1, l}^{Q}=\dot{E}_{l}$.

\section{Interlayer thermal current for layered Kitaev honeycomb model}

For the $Z_{2}$ system, the coupling term is given by Eq. (A8),

$$
\begin{aligned}
H_{\perp}= & \frac{1}{2} J_{\perp} F_{0} \sum_{l, l^{\prime}=l \pm 1} \int \frac{d^{2} k}{(2 \pi)^{2}} \frac{d^{2} k^{\prime}}{(2 \pi)^{2}} \frac{d^{2} q}{(2 \pi)^{2}} \\
& \times\left[\psi_{l}^{A \dagger}(\mathbf{k}) \psi_{l}^{A}(\mathbf{k}+\mathbf{q}) \psi_{l^{\prime}}^{A \dagger}\left(\mathbf{k}^{\prime}\right) \psi_{l^{\prime}}^{A}\left(\mathbf{k}^{\prime}-\mathbf{q}\right)+\left(A^{l} \rightarrow B^{l}\right)+\left(A^{l^{\prime}} \rightarrow B^{l^{\prime}}\right)+\left(A^{l} \rightarrow B^{l} \& A^{l^{\prime}} \rightarrow B^{l^{\prime}}\right)\right],
\end{aligned}
$$

and therefore,

$$
\begin{aligned}
J^{Q}= & \frac{1}{2} J_{\perp} F_{0} \int \frac{d^{2} k}{(2 \pi)^{2}} \frac{d^{2} k^{\prime}}{(2 \pi)^{2}} \frac{d^{2} q}{(2 \pi)^{2}} \sum_{l} \partial_{t}\left[\psi_{l}^{A \dagger}(\mathbf{k}) \psi_{l}^{A}(\mathbf{k}+\mathbf{q})\right] \\
& \times\left[\left(\psi_{l+1}^{A \dagger}\left(\mathbf{k}^{\prime}\right) \psi_{l+1}^{A}\left(\mathbf{k}^{\prime}-\mathbf{q}\right)-\psi_{l-1}^{A \dagger}\left(\mathbf{k}^{\prime}\right) \psi_{l-1}^{A}\left(\mathbf{k}^{\prime}-\mathbf{q}\right)\right)+\left(A^{l} \rightarrow B^{l}\right)+\left(A^{l^{\prime}} \rightarrow B^{l^{\prime}}\right)+\left(A^{l} \rightarrow B^{l} \& A^{l^{\prime}} \rightarrow B^{l^{\prime}}\right)\right] .
\end{aligned}
$$

Fourier transforming with respect to imaginary time results in

$$
\begin{aligned}
J^{Q}\left(i \omega_{n}\right)= & \frac{1}{2} J_{\perp} F_{0} \int \frac{d^{2} k}{(2 \pi)^{2}} \frac{d^{2} k^{\prime}}{(2 \pi)^{2}} \frac{d^{2} q}{(2 \pi)^{2}} \frac{1}{\beta^{3}} \sum_{\nu_{n}, \nu_{m}, \Omega_{n}} \Omega_{n} \sum_{l}\left[\psi_{l}^{A \dagger}\left(\mathbf{k}, i \nu_{n}\right) \psi_{l}^{A}\left(\mathbf{k}+\mathbf{q}, i \nu_{n}+i \Omega_{n}\right)\right] \\
& \times\left[\left(\psi_{l+1}^{A \dagger}\left(\mathbf{k}^{\prime}, i \nu_{m}\right) \psi_{l+1}^{A}\left(\mathbf{k}^{\prime}-\mathbf{q}, i \nu_{m}-i \Omega_{n}+i \omega_{n}\right)-(l+1) \rightarrow(l-1)\right)\right. \\
& \left.+\left(A^{l} \rightarrow B^{l}\right)+\left(A^{l^{\prime}} \rightarrow B^{l^{\prime}}\right)+\left(A^{l} \rightarrow B^{l} \& A^{l^{\prime}} \rightarrow B^{l^{\prime}}\right)\right] .
\end{aligned}
$$

We again revert to the complex fermion representation and consider states only near the Dirac point $\mathbf{K}$. Transforming to the eigenstates of $H$ yields, for $m=0$,

$$
\begin{aligned}
J^{Q}\left(i \omega_{n}\right)= & \frac{1}{2^{5}} J_{\perp} \sum_{l, \eta= \pm 1} \eta \int \frac{d^{2} k}{(2 \pi)^{2}} \frac{d^{2} k^{\prime}}{(2 \pi)^{2}} \frac{d^{2} q}{(2 \pi)^{2}} \sum_{\lambda_{1} \ldots \lambda_{4}= \pm 1} \frac{1}{\beta^{3}} \sum_{\nu_{n}, \nu_{m}, \Omega_{n}} \Omega_{n} \\
& \times F_{\mathbf{k}, \mathbf{k}^{\prime}, \mathbf{q}}^{\lambda_{1} \ldots \lambda_{4}} a_{\lambda_{1}}^{l \dagger}\left(\mathbf{k}, i \nu_{n}\right) a_{\lambda_{2}}^{l}\left(\mathbf{k}+\mathbf{q}, i \nu_{n}+i \Omega_{n}+i \omega_{n}\right) a_{\lambda_{3}}^{l+\eta^{\dagger}}\left(\mathbf{k}^{\prime}, i \nu_{m}\right) a_{\lambda_{4}}^{l+\eta}\left(\mathbf{k}^{\prime}-\mathbf{q}, i \nu_{m}-i \Omega_{n}\right),
\end{aligned}
$$

where $F_{\mathbf{k}, \mathbf{k}^{\prime}, \mathbf{q}}^{\lambda_{1} \ldots \lambda_{4}}$ corresponds to the transformation of $F_{0}$ from the sublattice to the eigenstate basis, while for the case $\Delta>m>0, m \gg \Delta-m$, we neglect the contribution of the $B$ sublattice and get a simpler expression, 


$$
\begin{aligned}
J^{Q}\left(i \omega_{n}\right)= & \frac{1}{2^{5}} J_{\perp} F_{0} \sum_{l, \eta= \pm 1} \eta \int \frac{d^{2} k}{(2 \pi)^{2}} \frac{d^{2} k^{\prime}}{(2 \pi)^{2}} \frac{d^{2} q}{(2 \pi)^{2}} \frac{1}{\beta^{3}} \sum_{\nu_{n}, \nu_{m}, \Omega_{n}} \Omega_{n} \\
& \times a^{l \dagger}\left(\mathbf{k}, i \nu_{n}\right) a^{l}\left(\mathbf{k}+\mathbf{q}, i \nu_{n}+i \Omega_{n}+i \omega_{n}\right) a^{l+\eta \dagger}\left(\mathbf{k}^{\prime}, i \nu_{m}\right) a^{l+\eta}\left(\mathbf{k}^{\prime}-\mathbf{q}, i \nu_{m}-i \Omega_{n}\right) .
\end{aligned}
$$

\section{Thermal conductivity}

The thermal conductivity is given by [74-76]

$$
\kappa=\frac{-1}{T} \lim _{\omega \rightarrow 0} \frac{\operatorname{Im}[\Pi(\omega)]}{\omega},
$$

where $\Pi(\omega)$ is the retarded thermal current-thermal current correlation function. Using Eq. (14), and an extended definition of the four-point correlation function in Eq. (15) (in the TR broken case, there is only a single band $\lambda$ that crosses the Fermi surface)

$$
\Upsilon_{\lambda_{1}, \lambda_{2}}\left(\mathbf{k}_{1}, \mathbf{k}_{1}{ }^{\prime}, \mathbf{q} ; i \nu_{n}, i \nu_{m}\right)=\left\langle a_{\lambda_{1}}^{\dagger}\left(\mathbf{k}_{1}, i \nu_{n}\right) a_{\lambda_{2}}\left(\mathbf{k}_{1}+\mathbf{q}, i \nu_{m}\right) a_{\lambda_{2}}^{\dagger}\left(\mathbf{k}_{1}^{\prime}+\mathbf{q}, i \nu_{m}\right) a_{\lambda_{1}}\left(\mathbf{k}_{1}{ }^{\prime}, i \nu_{n}\right)\right\rangle
$$

we get

$$
\begin{aligned}
\Pi\left(i \omega_{n}\right)= & \frac{1}{64} J_{\perp}^{2} \frac{1}{\beta} \sum_{\Omega_{n}} \Omega_{n}^{2} \int \frac{d^{2} q}{(2 \pi)^{2}} \frac{1}{\beta} \sum_{\nu_{n}} \int \frac{d^{2} k_{1} d^{2} k_{2}}{(2 \pi)^{4}} \frac{1}{\beta} \sum_{\nu_{m}} \int \frac{d^{2} k_{1}^{\prime} d^{2} k_{2}^{\prime}}{(2 \pi)^{4}} \\
& \times \sum_{\lambda_{1} \ldots \lambda_{4}} F_{\mathbf{k}_{1}, \mathbf{k}_{\mathbf{1}}^{\prime}, \mathbf{q}}^{\lambda_{1}, \lambda_{2}, \lambda_{3}, \lambda_{4}} F_{\mathbf{k}_{\mathbf{2}}, \mathbf{k}_{2}^{\prime}, \mathbf{q}}^{\lambda_{2}, \lambda_{1}, \lambda_{4}, \lambda_{3}} \Upsilon_{\lambda_{2}, \lambda_{1}}\left(\mathbf{k}_{\mathbf{1}}, \mathbf{k}_{\mathbf{2}}, \mathbf{q} ;-i \nu_{n}-i \Omega_{n}-i \omega_{n},-i \nu_{n}\right) \Upsilon_{\lambda_{3}, \lambda_{4}}\left(\mathbf{k}_{\mathbf{1}}^{\prime}, \mathbf{k}_{\mathbf{2}}^{\prime},-\mathbf{q} ; i \nu_{m}, i \nu_{m}-i \Omega_{n}\right) .
\end{aligned}
$$

The function $\Upsilon_{\mathbf{k}}\left(z, z+i \Omega_{n}\right)$ (suppressing the momentum and $\lambda$ dependence for clarity) has branch cuts for $\operatorname{Im}[z]=0, \operatorname{Im}[z]=-i \Omega_{n}$; using the usual contour integration method, as explained in Ref. [93], for example, it can be shown that

$$
\begin{aligned}
& \frac{1}{\beta} \sum_{\nu_{n}} \Upsilon\left(z, z+i \Omega_{n}\right) \\
& \quad=\int \frac{d \epsilon_{1}}{2 \pi i} n_{F}\left(\epsilon_{1}\right)\left[\Upsilon_{\mathbf{k}}\left(\epsilon_{1}^{+}, \epsilon_{1}+i \Omega_{n}\right)-\Upsilon_{\mathbf{k}}\left(\epsilon_{1}^{-}, \epsilon_{1}+i \Omega_{n}\right)\right]+\int \frac{d \epsilon_{1}}{2 \pi i} n_{F}\left(\epsilon_{1}\right)\left[\Upsilon_{\mathbf{k}}\left(\epsilon_{1}-i \Omega_{n}, \epsilon_{1}^{+}\right)-\Upsilon_{\mathbf{k}}\left(\epsilon_{1}-i \Omega_{n}, \epsilon_{1}^{-}\right)\right]
\end{aligned}
$$

where $\epsilon^{ \pm}=\epsilon \pm i \delta$, with $\delta$ a positive infinitesimal. By first performing the summations over $\nu_{n}$ and $\nu_{m}$, which result in integrations over $\epsilon_{1}, \epsilon_{3}$, the summation over the bosonic frequencies $\Omega_{n}$ gives (again integrating along the branch cuts of the $\Upsilon$ functions).

$$
\begin{aligned}
Q\left(i \omega_{n}\right) \equiv & \frac{1}{\beta^{3}} \sum_{\nu_{n}, \nu_{m}, \Omega_{n}} \Omega_{n}^{2} \Upsilon_{\mathbf{k}}\left(i \nu_{n}, i \nu_{n}+i \Omega_{n}\right) \Upsilon_{\mathbf{k}^{\prime}}\left(i \nu_{m}, i \nu_{m}-i \Omega_{n}+i \omega_{n}\right) \\
= & \int \frac{d \epsilon_{1}}{2 \pi i} \int \frac{d \epsilon_{3}}{2 \pi i} n_{F}\left(\epsilon_{1}\right) n_{F}\left(\epsilon_{3}\right) \int \frac{d \epsilon_{2}}{2 \pi i} n_{B}(x) x^{2}\left[\Upsilon_{\mathbf{k}}\left(\epsilon_{1}^{+}, \epsilon_{2}^{+}\right)-\Upsilon_{\mathbf{k}}\left(\epsilon_{1}^{+}, \epsilon_{2}^{-}\right)-\Upsilon_{\mathbf{k}}\left(\epsilon_{1}^{-}, \epsilon_{2}^{+}\right)\right. \\
& \left.+\Upsilon_{\mathbf{k}}\left(\epsilon_{1}^{-}, \epsilon_{2}^{-}\right)\right]\left[\Upsilon_{\mathbf{k}^{\prime}}\left(\epsilon_{3}^{+},-x+\epsilon_{3}+i \omega_{n}\right)-\Upsilon_{\mathbf{k}^{\prime}}\left(\epsilon_{3}^{-},-x+\epsilon_{3}+i \omega_{n}\right)+\Upsilon_{\mathbf{k}^{\prime}}\left(x+\epsilon_{3}-i \omega_{n}, \epsilon_{3}^{+}\right)\right. \\
& \left.-\Upsilon_{\mathbf{k}^{\prime}}\left(x+\epsilon_{3}-i \omega_{n}, \epsilon_{3}^{-}\right)\right]-n_{B}(-x) x^{2}\left[\Upsilon_{\mathbf{k}}\left(\epsilon_{2}^{+}, \epsilon_{1}^{+}\right)-\Upsilon_{\mathbf{k}}\left(\epsilon_{2}^{+}, \epsilon_{1}^{-}\right)-\Upsilon_{\mathbf{k}}\left(\epsilon_{2}^{-}, \epsilon_{1}^{+}\right)\right. \\
& \left.+\Upsilon_{\mathbf{k}}\left(\epsilon_{2}^{-}, \epsilon_{1}^{-}\right)\right]\left[\Upsilon_{\mathbf{k}^{\prime}}\left(\epsilon_{3}^{+}, x+\epsilon_{3}+i \omega_{n}\right)-\Upsilon_{\mathbf{k}^{\prime}}\left(\epsilon_{3}^{-}, x+\epsilon_{3}+i \omega_{n}\right)+\Upsilon_{\mathbf{k}^{\prime}}\left(-x+\epsilon_{3}-i \omega_{n}, \epsilon_{3}^{+}\right)\right. \\
& \left.-\Upsilon_{\mathbf{k}^{\prime}}\left(-x+\epsilon_{3}-i \omega_{n}, \epsilon_{3}^{-}\right)\right]+n_{B}(y)\left(y+i \omega_{n}\right)^{2}\left[\Upsilon_{\mathbf{k}^{\prime}}\left(\epsilon_{2}^{+}, \epsilon_{3}^{+}\right)-\Upsilon_{\mathbf{k}^{\prime}}\left(\epsilon_{2}^{+}, \epsilon_{3}^{-}\right)-\Upsilon_{\mathbf{k}^{\prime}}\left(\epsilon_{2}^{-}, \epsilon_{3}^{+}\right)\right. \\
& \left.+\Upsilon_{\mathbf{k}^{\prime}}\left(\epsilon_{2}^{-}, \epsilon_{3}^{-}\right)\right]\left[\Upsilon_{\mathbf{k}}\left(\epsilon_{1}^{+}, \epsilon_{1}+y+i \omega_{n}\right)-\Upsilon_{\mathbf{k}}\left(\epsilon_{1}^{-}, \epsilon_{1}+y+i \omega_{n}\right)+\Upsilon_{\mathbf{k}}\left(\epsilon_{1}-y-i \omega_{n}, \epsilon_{1}^{+}\right)\right. \\
& \left.-\Upsilon_{\mathbf{k}}\left(\epsilon_{1}-y-i \omega_{n}, \epsilon_{1}^{-}\right)\right]-n_{B}(-y)\left(-y+i \omega_{n}\right)^{2}\left[\Upsilon_{\mathbf{k}^{\prime}}\left(\epsilon_{3}^{+}, \epsilon_{2}^{+}\right)-\Upsilon_{\mathbf{k}^{\prime}}\left(\epsilon_{3}^{+}, \epsilon_{2}^{-}\right)-\Upsilon_{\mathbf{k}^{\prime}}\left(\epsilon_{3}^{-}, \epsilon_{2}^{+}\right)\right. \\
& \left.+\Upsilon_{\mathbf{k}^{\prime}}\left(\epsilon_{3}^{-}, \epsilon_{2}^{-}\right)\right]\left[\Upsilon_{\mathbf{k}}\left(\epsilon_{1}^{+}, \epsilon_{1}-y+i \omega_{n}\right)-\Upsilon_{\mathbf{k}}\left(\epsilon_{1}^{-}, \epsilon_{1}-y+i \omega_{n}\right)+\Upsilon_{\mathbf{k}}\left(\epsilon_{1}+y-i \omega_{n}, \epsilon_{1}^{+}\right)\right. \\
& \left.-\Upsilon_{\mathbf{k}}\left(\epsilon_{1}+y-i \omega_{n}, \epsilon_{1}^{-}\right)\right]
\end{aligned}
$$

with $x=\epsilon_{2}-\epsilon_{1}, y=\epsilon_{2}-\epsilon_{3}$. 
Performing the analytical continuation by replacing $i \omega_{n} \rightarrow \omega+i \delta$ and a few further manipulations result in

$$
\begin{aligned}
Q(\omega)= & \int \frac{d \epsilon_{1}}{2 \pi i} \int \frac{d \epsilon_{3}}{2 \pi i} \int \frac{d \epsilon_{2}}{2 \pi i} n_{B}(x) x^{2}\left[n_{F}\left(\epsilon_{1}\right)-n_{F}\left(\epsilon_{2}\right)\right]\left[\Upsilon_{\mathbf{k}}\left(\epsilon_{1}^{+}, \epsilon_{2}^{+}\right)-\Upsilon_{\mathbf{k}}\left(\epsilon_{1}^{+}, \epsilon_{2}^{-}\right)+\text {c.c. }\right]\left[n _ { F } ( \epsilon _ { 3 } ) \left(\Upsilon_{\mathbf{k}^{\prime}}\left(\epsilon_{3}^{+}, \epsilon_{3}^{+}-x+\omega\right)\right.\right. \\
& \left.\left.-\Upsilon_{\mathbf{k}^{\prime}}\left(\epsilon_{3}^{-}, \epsilon_{3}^{+}-x+\omega\right)\right)+n_{F}\left(\epsilon_{3}-x+\omega\right)\left(\Upsilon_{\mathbf{k}^{\prime}}\left(\epsilon_{3}^{-}, \epsilon_{3}^{+}-x+\omega\right)-\Upsilon_{\mathbf{k}^{\prime}}\left(\epsilon_{3}^{-}, \epsilon_{3}^{-}-x+\omega\right)\right)\right] \\
& -n_{B}(y)(-y+\omega)^{2}\left[n_{F}\left(\epsilon_{3}\right)-n_{F}\left(\epsilon_{2}\right)\right]\left[\Upsilon_{\mathbf{k}^{\prime}}\left(\epsilon_{3}^{+}, \epsilon_{2}^{+}\right)-\Upsilon_{\mathbf{k}^{\prime}}\left(\epsilon_{3}^{-}, \epsilon_{2}^{+}\right)-c . c\right]\left[n _ { F } ( \epsilon _ { 1 } ) \left(\Upsilon_{\mathbf{k}}\left(\epsilon_{1}^{+}, \epsilon_{1}^{+}-y+\omega\right)\right.\right. \\
& \left.\left.-\Upsilon_{\mathbf{k}}\left(\epsilon_{1}^{-}, \epsilon_{1}-y+\omega\right)\right)+n_{F}\left(\epsilon_{1}-y+\omega\right)\left(\Upsilon_{\mathbf{k}}\left(\epsilon_{1}^{-}, \epsilon_{1}^{+}-y+\omega\right)-\Upsilon_{\mathbf{k}}\left(\epsilon_{1}^{-}, \epsilon_{1}^{-}-y+\omega\right)\right)\right] .
\end{aligned}
$$

The imaginary part of the above expression is [using the fact that $\Upsilon_{\mathbf{k}}\left(\epsilon_{1}^{+}, \epsilon_{2}^{+}\right)=\Upsilon_{\mathbf{k}}\left(\epsilon_{1}^{-}, \epsilon_{2}^{-}\right)^{*}$ and $\Upsilon_{\mathbf{k}}\left(\epsilon_{1}^{+}, \epsilon_{2}^{-}\right)=$ $\left.\Upsilon_{\mathbf{k}}\left(\epsilon_{1}^{-}, \epsilon_{2}^{+}\right)^{*}\right]$

$$
\begin{aligned}
\Im[Q(\omega)]= & \int \frac{d \epsilon_{1}}{2 \pi i} \int \frac{d \epsilon_{3}}{2 \pi i} \int \frac{d \epsilon_{2}}{2 \pi i} 2 \operatorname{Re}\left[\Upsilon_{\mathbf{k}}\left(\epsilon_{1}^{+}, \epsilon_{2}^{+}\right)-\Upsilon_{\mathbf{k}}\left(\epsilon_{1}^{+}, \epsilon_{2}^{-}\right)\right] \times \operatorname{Re}\left[\Upsilon_{\mathbf{k}^{\prime}}\left(\epsilon_{3}^{+}, \epsilon_{3}^{+}-x+\omega\right)\right. \\
& \left.-\Upsilon_{\mathbf{k}^{\prime}}\left(\epsilon_{3}^{-}, \epsilon_{3}^{+}-x+\omega\right)\right]\left[n_{F}\left(\epsilon_{3}\right)-n_{F}\left(\epsilon_{3}-x+\omega\right)\right] \times\left[n_{F}\left(\epsilon_{1}\right)-n_{F}\left(\epsilon_{2}\right)\right] n_{B}(x) x^{2} \\
& -2 \operatorname{Re}\left[\Upsilon_{\mathbf{k}^{\prime}}\left(\epsilon_{3}^{+}, \epsilon_{2}^{+}\right)-\Upsilon_{\mathbf{k}^{\prime}}\left(\epsilon_{3}^{-}, \epsilon_{2}^{+}\right)\right] \times \operatorname{Re}\left[\Upsilon_{\mathbf{k}}\left(\epsilon_{1}^{+}, \epsilon_{1}^{+}-y+\omega\right)\right. \\
& \left.-\Upsilon_{\mathbf{k}}\left(\epsilon_{1}^{-}, \epsilon_{1}^{+}-y+\omega\right)\right]\left[n_{F}\left(\epsilon_{1}\right)-n_{F}\left(\epsilon_{1}-y+\omega\right)\right]\left[n_{F}\left(\epsilon_{1}\right)-n_{F}\left(\epsilon_{2}\right)\right] n_{B}(-y)(-y+\omega)^{2} .
\end{aligned}
$$

In the second line, we replace $\epsilon_{2} \rightarrow \epsilon_{1}-\epsilon_{2}+\epsilon_{3}+\omega$ to get

$$
\begin{aligned}
\Im[Q(\omega)]= & \int \frac{d \epsilon_{1}}{2 \pi i} \int \frac{d \epsilon_{3}}{2 \pi i} \int \frac{d \epsilon_{2}}{2 \pi i} 2 \operatorname{Re}\left[\Upsilon_{\mathbf{k}}\left(\epsilon_{1}^{+}, \epsilon_{2}^{+}\right)-\Upsilon_{\mathbf{k}}\left(\epsilon_{1}^{+}, \epsilon_{2}^{-}\right)\right] \times \operatorname{Re}\left[\Upsilon_{\mathbf{k}^{\prime}}\left(\epsilon_{3}^{+}, \epsilon_{1}-\epsilon_{2}+\epsilon_{3}^{+}+\omega\right)\right. \\
& \left.-\Upsilon_{\mathbf{k}^{\prime}}\left(\epsilon_{3}^{-}, \epsilon_{1}-\epsilon_{2}+\epsilon_{3}^{+}+\omega\right)\right]\left(\epsilon_{2}-\epsilon_{1}\right)^{2}\left[n_{F}\left(\epsilon_{1}\right)-n_{F}\left(\epsilon_{2}\right)\right]\left[n_{F}\left(\epsilon_{3}\right)-n_{F}\left(\epsilon_{3}-\epsilon_{2}+\epsilon_{1}+\omega\right)\right]\left[n_{B}\left(\epsilon_{2}-\epsilon_{1}\right)\right. \\
& \left.-n_{B}\left(\epsilon_{2}-\epsilon_{1}-\omega\right)\right] .
\end{aligned}
$$

Therefore, using the identity

$\left[n_{F}\left(\epsilon_{1}\right)-n_{F}\left(\epsilon_{2}\right)\right]\left[n_{F}\left(\epsilon_{3}\right)-n_{F}\left(\epsilon_{3}-\epsilon_{2}+\epsilon_{1}\right)\right] \frac{\partial}{\partial \epsilon} n_{B}\left(\epsilon_{2}-\epsilon_{1}\right)=\frac{1}{T}\left(1-n_{F}\left(\epsilon_{1}\right)\right) n_{F}\left(\epsilon_{2}\right)\left(1-n_{F}\left(\epsilon_{3}\right)\right) n_{F}\left(\epsilon_{1}-\epsilon_{2}+\epsilon_{3}\right)$,

we get

$$
\begin{aligned}
\lim _{\omega \rightarrow 0} \frac{\operatorname{Im}[Q(\omega)]}{\omega}= & \frac{1}{T} \int \frac{d \epsilon_{1}}{2 \pi i} \int \frac{d \epsilon_{3}}{2 \pi i} \int \frac{d \epsilon_{2}}{2 \pi i} 2 \operatorname{Re}\left[\Upsilon_{\mathbf{k}}\left(\epsilon_{1}^{+}, \epsilon_{2}^{+}\right)-\Upsilon_{\mathbf{k}}\left(\epsilon_{1}^{+}, \epsilon_{2}^{-}\right)\right] \times \operatorname{Re}\left[\Upsilon_{\mathbf{k}^{\prime}}\left(\epsilon_{3}^{+}, \epsilon_{1}-\epsilon_{2}+\epsilon_{3}^{+}+\omega\right)\right. \\
& \left.-\Upsilon_{\mathbf{k}^{\prime}}\left(\epsilon_{3}^{-}, \epsilon_{1}-\epsilon_{2}+\epsilon_{3}^{+}+\omega\right)\right]\left(\epsilon_{2}-\epsilon_{1}\right)^{2} \times\left(1-n_{F}\left(\epsilon_{1}\right)\right) n_{F}\left(\epsilon_{2}\right)\left(1-n_{F}\left(\epsilon_{3}\right)\right) n_{F}\left(\epsilon_{1}-\epsilon_{2}+\epsilon_{3}\right) .
\end{aligned}
$$

Inserting this into the formula for $\kappa$, Eq. (12), and writing the momentum and band dependence explicitly, results in

$$
\begin{aligned}
\kappa \sim & \frac{J_{\perp}^{2}}{T^{2}} \int \frac{d^{2} k_{1}}{(2 \pi)^{2}} \frac{d^{2} k_{2}}{(2 \pi)^{2}} \frac{d^{2} k_{1}^{\prime}}{(2 \pi)^{2}} \frac{d^{2} k_{2}^{\prime}}{(2 \pi)^{2}} \frac{d^{2} q}{(2 \pi)^{2}} \int \frac{d \epsilon_{1}}{2 \pi} \int \frac{d \epsilon_{2}}{2 \pi} \int \frac{d \epsilon_{3}}{2 \pi} \sum_{\lambda_{1} \ldots 4} F_{\mathbf{k}_{1}, \mathbf{k}_{1}^{\prime}, \mathbf{q}}^{\lambda_{1}, \lambda_{1}, \lambda_{3}, \lambda_{4}} F_{\mathbf{k}_{2}, \mathbf{k}_{2}^{\prime},-\mathbf{q}}^{\lambda_{2}, \lambda_{1}, \lambda_{4}, \lambda_{3}} \operatorname{Re}\left[\Upsilon_{\lambda_{1} \lambda_{2}}\left(\mathbf{k}_{\mathbf{1}}, \mathbf{\mathbf { k } _ { 2 }}, \mathbf{q} ; \epsilon_{1}^{+}, \epsilon_{2}^{+}\right)\right. \\
& \left.-\Upsilon_{\lambda_{1} \lambda_{2}}\left(\mathbf{k}_{\mathbf{1}}, \mathbf{k}_{\mathbf{2}}, \mathbf{q} ; \epsilon_{1}^{+}, \epsilon_{2}^{-}\right)\right] \operatorname{Re}\left[\Upsilon_{\lambda_{3} \lambda_{4}}\left(\mathbf{k}_{1}^{\prime}, \mathbf{k}_{\mathbf{2}}^{\prime},-\mathbf{q} ; \epsilon_{3}^{+}, \epsilon_{1}-\epsilon_{2}+\epsilon_{3}^{+}\right)\right. \\
& \left.-\Upsilon_{\lambda_{3} \lambda_{4}}\left(\mathbf{k}_{\mathbf{1}}^{\prime}, \mathbf{k}_{2}^{\prime},-\mathbf{q} ; \epsilon_{3}^{-}, \epsilon_{1}-\epsilon_{2}+\epsilon_{3}^{+}\right)\right]\left(1-n_{F}\left(\epsilon_{1}\right)\right) n_{F}\left(\epsilon_{2}\right)\left(1-n_{F}\left(\epsilon_{3}\right)\right) n_{F}\left(\epsilon_{1}-\epsilon_{2}+\epsilon_{3}\right) \times\left(\epsilon_{1}-\epsilon_{2}\right)^{2} .
\end{aligned}
$$

\section{Clean case}

In this case, as $\Upsilon_{\lambda_{1}, \lambda_{2}}\left(\mathbf{k}_{\mathbf{1}}, \mathbf{k}_{\mathbf{2}}, \mathbf{q} ; \epsilon_{1}^{ \pm}, \epsilon_{2}^{+}\right)=\delta\left(\mathbf{k}_{\mathbf{1}}-\mathbf{k}_{\mathbf{2}}\right) G_{\lambda_{1}}^{R / A}\left(\mathbf{k}_{\mathbf{1}}, \epsilon_{1}\right) G_{\lambda_{2}}^{R}\left(\mathbf{k}_{\mathbf{1}}+\mathbf{q}, \epsilon_{2}\right)$, the formula for the thermal conductivity is

$$
\begin{aligned}
\kappa= & \frac{J_{\perp}^{2}}{T^{2}} \int \frac{d^{2} k}{(2 \pi)^{2}} \frac{d^{2} k^{\prime}}{(2 \pi)^{2}} \frac{d^{2} q}{(2 \pi)^{2}} \int \frac{d \epsilon_{1}}{2 \pi} \int \frac{d \epsilon_{2}}{2 \pi} \int \frac{d \epsilon_{3}}{2 \pi}\left(\epsilon_{1}-\epsilon_{2}\right)^{2} \sum_{\lambda_{1} \ldots 4}\left|F_{\mathbf{k}, \mathbf{k}^{\prime}, \mathbf{q}}^{\lambda_{1}, \lambda_{2}, \lambda_{4}, \lambda_{4}}\right|^{2} \\
& \times A_{\lambda_{1}}\left(\mathbf{k}, \epsilon_{1}\right) A_{\lambda_{2}}\left(\mathbf{k}+\mathbf{q}, \epsilon_{2}\right) A_{\lambda_{3}}\left(\mathbf{k}^{\prime}, \epsilon_{3}\right) A_{\lambda_{4}}\left(\mathbf{k}^{\prime}-\mathbf{q}, \epsilon_{1}-\epsilon_{2}+\epsilon_{3}\right)\left(1-n_{F}\left(\epsilon_{1}\right)\right) n_{F}\left(\epsilon_{2}\right)\left(1-n_{F}\left(\epsilon_{3}\right)\right) n_{F}\left(\epsilon_{1}-\epsilon_{2}+\epsilon_{3}\right),
\end{aligned}
$$


with $A_{\lambda}(\mathbf{k}, \epsilon)=-2 \operatorname{Im}\left[G_{\lambda}^{R}(\mathbf{k}, \epsilon)\right]$ the spinon spectral function, which is $A_{\lambda}\left(\mathbf{k}, \epsilon_{1}\right)=2 \pi \delta\left(\epsilon-\epsilon_{\mathbf{k}}^{\lambda}\right)$ in the clean case. This results in the formula derived in the main text, Eq. (4).

\section{Effects of potential disorder}

For a $Z_{2}$ QSL with a Fermi surface, we consider the effects of potential disorder. In the self-consistent Born approximation (SCBA), which is valid for weak disorder such that $k_{F} \ell \gg 1$, the dressed Green's function has the form

$$
G_{\lambda}^{R}(\mathbf{k}, \omega)=G_{\lambda}^{A *}(\mathbf{k}, \omega)=\frac{1}{\omega-\epsilon_{\lambda \mathbf{k}}+i / 2 \tau},
$$

where $\tau=\ell / v_{F}$ is the disorder-induced lifetime.

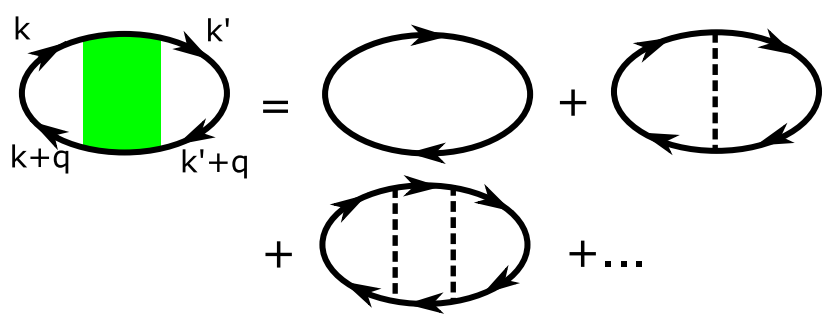

FIG. 5. In the self-consistent Born approximation, which is valid when $k_{F} l_{m f p} \gg 1$, only ladder diagrams without crossed disorder lines contribute to the vertex function. In this figure, the solid lines are renormalized electron propagators, and dashed lines represent the effects of disorder.

In calculating the four-point correlation function

$$
\begin{aligned}
\Upsilon\left(\mathbf{k}, \mathbf{k}^{\prime}, \mathbf{q} ; i \nu_{n}, i \nu_{m}\right)= & \left\langle\psi_{l}^{\dagger}\left(\mathbf{k}, i \nu_{n}\right) \psi_{l}\left(\mathbf{k}+\mathbf{q}, i \nu_{m}\right) \psi_{l}^{\dagger}\right. \\
& \left.\times\left(\mathbf{k}^{\prime}+\mathbf{q}, i \nu_{m}\right) \psi_{l}\left(\mathbf{k}^{\prime}, i \nu_{n}\right)\right\rangle_{\mathrm{dis}},
\end{aligned}
$$

we define the vertex function $\Gamma_{\mathbf{q}}\left(i \nu_{n}, i \nu_{m}\right)$ such that

$\Upsilon\left(\mathbf{k}, \mathbf{k}^{\prime}, \mathbf{q} ; i \nu_{n}, i \nu_{m}\right)=\delta\left(\mathbf{k}-\mathbf{k}^{\prime}\right) G\left(\mathbf{k}, i \nu_{n}\right) G\left(\mathbf{k}+\mathbf{q}, i \nu_{m}\right)+\Gamma_{\mathbf{q}}\left(i \nu_{n}, i \nu_{m}\right) G\left(\mathbf{k}, i \nu_{n}\right) G\left(\mathbf{k}+\mathbf{q}, i \nu_{m}\right) G\left(\mathbf{k}^{\prime}, i \nu_{n}\right) G\left(\mathbf{k}^{\prime}+\mathbf{q}, i \nu_{m}\right)$.

In the SCBA, the vertex function is given by the set of ladder diagrams, which are schematically shown in Fig. 5. The sum of all ladder diagrams results in the following self-consistent equation for $\Gamma_{\mathbf{q}}\left(i \nu_{n}, i \nu_{m}\right)$ :

$$
\begin{aligned}
\Gamma_{\lambda, \lambda}\left(\mathbf{k}, \mathbf{k}+\mathbf{q} ; i \nu_{n}, i \nu_{m}\right) & =\frac{1}{2 \pi \nu \tau}+\frac{1}{2 \pi \nu \tau} \Gamma_{\mathbf{q}}\left(i \nu_{n}, i \nu_{m}\right) \int \frac{d^{2} k}{(2 \pi)^{2}} G\left(\mathbf{k}, i \nu_{n}\right) G\left(\mathbf{k}+\mathbf{q}, i \nu_{m}\right) \\
& =\frac{1}{2 \pi \nu \tau} \frac{1}{1-\frac{1}{2 \pi \nu \tau} \int \frac{d^{2} k}{(2 \pi)^{2}} G\left(\mathbf{k}, i \nu_{n}\right) G\left(\mathbf{k}+\mathbf{q}, i \nu_{m}\right)}
\end{aligned}
$$

The important contribution to the thermal conductivity comes from the region of small $\mathbf{q}$ and small frequencies $\left(\epsilon, v_{F} \mathbf{q}<T\right.$, with $v_{F}$ the Fermi velocity). In this region,

$$
\Gamma\left(\mathbf{q} ; \epsilon_{1}^{+}, \epsilon_{2}^{-}\right) \approx \frac{1}{2 \pi \nu \tau^{2}} \frac{1}{-i\left(\epsilon_{2}-\epsilon_{1}\right)+D q^{2}}, \quad \Gamma\left(\mathbf{q} ; \epsilon_{1}^{+}, \epsilon_{2}^{+}\right) \approx 0
$$

with $D=v l_{m f p} / 2$ the diffusion constant.

Starting from Eq. (D20), we perform the Sommerfeld expansion with respect to $\epsilon_{1}$. The first nonvanishing contribution, in powers of $T$, occurs for the term

$$
\begin{aligned}
\kappa \sim & J_{\perp}^{2} \int \frac{d^{2} k_{1}}{(2 \pi)^{2}} \frac{d^{2} k_{2}}{(2 \pi)^{2}} \frac{d^{2} k_{1}^{\prime}}{(2 \pi)^{2}} \frac{d^{2} k_{2}^{\prime}}{(2 \pi)^{2}} \frac{d^{2} q}{(2 \pi)^{2}} \int \frac{d \epsilon_{2}}{2 \pi} \int \frac{d \epsilon_{3}}{2 \pi} \operatorname{Re}\left[\Upsilon\left(\mathbf{k}_{\mathbf{1}}, \mathbf{k}_{\mathbf{2}}, \mathbf{q} ; 0^{+}, \epsilon_{2}^{+}\right)-\Upsilon\left(\mathbf{k}_{\mathbf{1}}, \mathbf{k}_{\mathbf{2}}, \mathbf{q} ; 0^{+}, \epsilon_{2}^{-}\right)\right] \\
& \times \operatorname{Re}\left[\Upsilon\left(\mathbf{k}_{\mathbf{1}}^{\prime}, \mathbf{k}_{\mathbf{2}}^{\prime},-\mathbf{q} ; \epsilon_{3}^{+},-\epsilon_{2}+\epsilon_{3}^{+}\right)-\Upsilon\left(\mathbf{k}_{\mathbf{1}}^{\prime}, \mathbf{k}_{\mathbf{2}}^{\prime},-\mathbf{q} ; \epsilon_{3}^{-},-\epsilon_{2}+\epsilon_{3}^{+}\right)\right] n_{F}\left(\epsilon_{2}\right)\left(1-n_{F}\left(\epsilon_{3}\right)\right) \partial_{\epsilon} n_{F}\left(-\epsilon_{2}+\epsilon_{3}\right) \times \epsilon_{2}^{2}
\end{aligned}
$$

where, at $T \ll E_{F}$, the last line becomes $n_{F}\left(\epsilon_{2}\right)\left(1-n_{F}\left(\epsilon_{3}\right)\right) \delta\left(-\epsilon_{2}+\epsilon_{3}\right) \times \epsilon_{2}^{2}=\delta\left(-\epsilon_{2}+\epsilon_{3}\right) T\left[\partial /\left(\partial \epsilon_{2}\right)\right] n_{F}\left(\epsilon_{2}\right) \epsilon_{2}^{2}$. Here, we have again set the form factor $\mathcal{F}_{\mathbf{k}, \mathbf{k}^{\prime}, \mathbf{q}}=1$ as we are dealing with a single band that crosses the Fermi energy, which is localized on the $A$ sublattice. 
After substituting the SCBA result, Eq. (D25), we are left with (neglecting terms with all poles on the same side of the real axis, as these give subleading contributions, and the vertexless terms, which give a $T^{3}$ result)

$$
\begin{aligned}
\kappa \sim & J_{\perp}^{2} T \int \frac{d^{2} k_{1}}{(2 \pi)^{2}} \frac{d^{2} k_{2}}{(2 \pi)^{2}} \frac{d^{2} k_{1}^{\prime}}{(2 \pi)^{2}} \frac{d^{2} k_{2}^{\prime}}{(2 \pi)^{2}} \frac{d^{2} q}{(2 \pi)^{2}} \int \frac{d \epsilon}{2 \pi} \Gamma_{\mathbf{q}}\left(0^{+}, \epsilon^{-}\right) G^{R}\left(\mathbf{k}_{\mathbf{1}}, 0\right) G^{A}\left(\mathbf{k}_{\mathbf{1}}, \epsilon\right) G^{R}\left(\mathbf{k}_{\mathbf{2}}, 0\right) G^{A}\left(\mathbf{k}_{\mathbf{2}}, \epsilon\right) \\
& \times \Gamma_{\mathbf{q}}\left(\epsilon^{+}, 0^{-}\right) G^{R}\left(\mathbf{k}_{\mathbf{1}}, \epsilon\right) G^{A}\left(\mathbf{k}_{\mathbf{1}}, 0\right) G^{R}\left(\mathbf{k}_{\mathbf{2}}, \epsilon\right) G^{A}\left(\mathbf{k}_{\mathbf{2}}, 0\right) \frac{\partial}{\partial \epsilon} n_{F}(\epsilon) \epsilon^{2} .
\end{aligned}
$$

We are interested in the contribution at small $\mathbf{q}$, which has the potential to be singular; we therefore set $\mathbf{q} \rightarrow 0$ and $\epsilon \rightarrow 0$ in the Green's functions, which results in [using the relation $G^{R}(\mathbf{k}, \epsilon) G^{A}(\mathbf{k}, \epsilon)=A^{2}(\mathbf{k}, \epsilon) \approx \tau \delta\left(\epsilon_{\mathbf{k}}-\epsilon\right)$, and therefore $\int d^{2} k /(2 \pi)^{2} G^{R}(\mathbf{k}, 0) G^{A}(\mathbf{k}, 0)=\nu \tau$, where $\nu$ is the density of states at the Fermi energy]

$$
\begin{aligned}
\kappa & \sim J_{\perp}^{2} T \int \frac{d \epsilon}{2 \pi} \int \frac{d^{2} q}{(2 \pi)^{2}} \operatorname{Re}\left[\frac{\nu}{-i \epsilon+D q^{2}}\right] \operatorname{Re}\left[\frac{\nu}{i \epsilon+D q^{2}}\right] \frac{\partial}{\partial \epsilon} n_{F}(\epsilon) \epsilon^{2} \\
& =J_{\perp}^{2} \nu^{2} T \int \frac{d \epsilon}{2 \pi} \int \frac{d^{2} q}{(2 \pi)^{2}}\left(\frac{D q^{2}}{\epsilon^{2}+D^{2} q^{4}}\right)^{2} \frac{\partial}{\partial \epsilon} n_{F}(\epsilon) \epsilon^{2} \\
& =J_{\perp}^{2} \frac{\nu^{2}}{D} T \int \frac{d \epsilon}{2 \pi} \frac{\partial}{\partial \epsilon} n_{F}(\epsilon)|\epsilon| \sim J_{\perp}^{2} \frac{\nu^{2}}{D} T^{2} .
\end{aligned}
$$

\section{Pair hopping term}

We have ignored the pair hopping term in the previous sections. This is because its contribution is similar to that of the spinon-hole hopping term. Performing the Matsubara summation for the pair hopping term results in

$$
\begin{aligned}
& \frac{J_{\perp}^{2}}{T^{2}} \int \frac{d^{2} k_{1}}{(2 \pi)^{2}} \frac{d^{2} k_{2}}{(2 \pi)^{2}} \frac{d^{2} k_{1}^{\prime}}{(2 \pi)^{2}} \frac{d^{2} k_{2}^{\prime}}{(2 \pi)^{2}} \frac{d^{2} q}{(2 \pi)^{2}} \int \frac{d \epsilon_{1}}{2 \pi} \int \frac{d \epsilon_{2}}{2 \pi} \int \frac{d \epsilon_{3}}{2 \pi} \sum_{\lambda_{1} \ldots 4} \\
& \quad \times \operatorname{Re}\left[\tilde{\Upsilon}_{\lambda_{1} \lambda_{2}}\left(\mathbf{k}_{\mathbf{1}}, \mathbf{k}_{\mathbf{2}}, \mathbf{q} ;-\epsilon_{1}^{+}, \epsilon_{2}^{+}\right)-\tilde{\Upsilon}_{\lambda_{1} \lambda_{2}}\left(\mathbf{k}_{\mathbf{1}}, \mathbf{\mathbf { k } _ { 2 }}, \mathbf{q} ;-\epsilon_{1}^{+}, \epsilon_{2}^{-}\right)\right] \\
& \quad \times \operatorname{Re}\left[\tilde{\Upsilon}_{\lambda_{3} \lambda_{4}}\left(\mathbf{k}_{\mathbf{1}}^{\prime}, \mathbf{k}_{\mathbf{2}}^{\prime},-\mathbf{q} ;-\epsilon_{3}^{+}, \epsilon_{1}-\epsilon_{2}+\epsilon_{3}^{+}\right)-\tilde{\Upsilon}_{\lambda_{3} \lambda_{4}}\left(\mathbf{k}_{\mathbf{1}}^{\prime}, \mathbf{k}_{\mathbf{2}}^{\prime},-\mathbf{q} ;-\epsilon_{3}^{-}, \epsilon_{1}-\epsilon_{2}+\epsilon_{3}^{+}\right)\right] \\
& \quad \times\left(1-n_{F}\left(\epsilon_{1}\right)\right) n_{F}\left(\epsilon_{2}\right)\left(1-n_{F}\left(\epsilon_{3}\right)\right) n_{F}\left(\epsilon_{1}-\epsilon_{2}+\epsilon_{3}\right) \times\left(\epsilon_{1}-\epsilon_{2}\right)^{2},
\end{aligned}
$$

where $\tilde{\Upsilon}_{\lambda_{1} \lambda_{2}}\left(\mathbf{k}_{\mathbf{1}}, \mathbf{k}_{\mathbf{2}}, \mathbf{q} ; i \nu_{n}, i \nu_{m}\right)=\left\langle a_{\lambda_{1}}\left(-\mathbf{k}_{\mathbf{1}},-i \nu_{n}\right) a_{\lambda_{2}}\left(\mathbf{k}_{\mathbf{1}}+\mathbf{q}, i \nu_{m}\right) a_{\lambda_{2}}^{\dagger}\left(\mathbf{k}_{\mathbf{2}}+\mathbf{q}, i \nu_{m}\right) a_{\lambda_{1}}^{\dagger}\left(-\mathbf{k}_{\mathbf{2}}, i \nu_{n}\right)\right\rangle$.

An analysis similar to that following Eq. (D23) shows that

$$
\begin{aligned}
\tilde{\Upsilon}_{\lambda_{1} \lambda_{2}}\left(\mathbf{k}_{\mathbf{1}}, \mathbf{k}_{\mathbf{2}}, \mathbf{q} ;-\epsilon_{1}^{+}, \epsilon_{2}^{-}\right)= & \delta\left(\mathbf{k}_{\mathbf{1}}-k_{\mathbf{2}}\right) G_{\lambda_{1}}^{R}\left(-\mathbf{k}_{\mathbf{1}}, \epsilon_{1}\right) G_{\lambda_{2}}^{A}\left(\mathbf{k}_{\mathbf{1}}+\mathbf{q}, \epsilon_{2}\right) \\
& +\Gamma_{\mathbf{q}}\left(\epsilon_{1}^{+}, \epsilon_{2}^{-}\right) G_{\lambda_{1}}^{R}\left(-\mathbf{k}_{\mathbf{1}}, \epsilon_{1}\right) G_{\lambda_{2}}^{A}\left(\mathbf{k}_{\mathbf{1}}+\mathbf{q}, \epsilon_{2}\right) G_{\lambda_{1}}^{R}\left(-\mathbf{k}_{\mathbf{2}}, \epsilon_{1}\right) G_{\lambda_{2}}^{A}\left(\mathbf{k}_{\mathbf{2}}+\mathbf{q}, \epsilon_{2}\right) \\
\tilde{\Upsilon}_{\lambda_{1} \lambda_{2}}\left(\mathbf{k}_{\mathbf{1}}, k_{\mathbf{2}}, \mathbf{q} ;-\epsilon_{1}^{+}, \epsilon_{2}^{+}\right)= & \delta\left(\mathbf{k}_{\mathbf{1}}-\mathbf{k}_{\mathbf{2}}\right) G_{\lambda_{1}}^{R}\left(-\mathbf{k}_{\mathbf{1}}, \epsilon_{1}\right) G_{\lambda_{2}}^{R}\left(\mathbf{k}_{\mathbf{1}}+\mathbf{q}, \epsilon_{2}\right),
\end{aligned}
$$

with $\Gamma_{\mathbf{q}}\left(\epsilon_{1}^{+}, \epsilon_{2}^{-}\right)=\left[1 /\left(2 \pi \nu \tau^{2}\right)\right]\left\{1 /\left[-i\left(\epsilon_{2}-\epsilon_{1}\right)+D q^{2}\right]\right\}$ as before; this term therefore contributes the same as the spinonhole hopping term.

\section{APPENDIX E: $U(1)$ QUANTUM SPIN LIQUID}

\section{Clean spinon thermal conductivity}

In this case, the interlayer coupling is given by Eq. (2),

$$
H_{\perp, \mathrm{sp}}=J_{\perp}^{\mathrm{sp}} \int \frac{d^{2} k d^{2} k^{\prime} d^{2} q}{(2 \pi)^{6}} \psi_{l}^{*}(\mathbf{k}) \psi_{l}(\mathbf{k}+\mathbf{q}) \psi_{l^{\prime}}^{*}\left(\mathbf{k}^{\prime}\right) \psi_{l^{\prime}}\left(\mathbf{k}^{\prime}-\mathbf{q}\right) .
$$

Plugging this into the formula for the thermal current operator Eq. (D6), and using the Kubo formula just as in Eq. (D14), results in 


$$
\begin{aligned}
\kappa_{\mathrm{sp}}= & \frac{J_{\perp}^{\mathrm{sp} 2}}{T^{2}} \int \frac{d^{2} k d^{2} k^{\prime} d^{2} q}{(2 \pi)^{6}} \int \frac{d \epsilon_{1} d \epsilon_{2} d \epsilon_{3}}{(2 \pi)^{3}} A\left(\mathbf{k}, \epsilon_{1}\right) A\left(\mathbf{k}+\mathbf{q}, \epsilon_{2}\right) A\left(\mathbf{k}^{\prime}, \epsilon_{3}\right) A\left(\mathbf{k}^{\prime}-\mathbf{q}, \epsilon_{1}-\epsilon_{2}+\epsilon_{3}\right) \\
& \times\left(\epsilon_{1}-\epsilon_{2}\right)^{2} n_{F}\left(-\epsilon_{1}\right) n_{F}\left(\epsilon_{2}\right) n_{F}\left(-\epsilon_{3}\right) n_{F}\left(\epsilon_{1}-\epsilon_{2}+\epsilon_{3}\right),
\end{aligned}
$$

with $A(\mathbf{k}, \epsilon)$ the spinon spectral function,

$$
A(\mathbf{k}, \epsilon)=\frac{2 c \epsilon^{2 / 3}}{\left(\epsilon_{\mathbf{k}}-\mu\right)^{2}+c^{2} \epsilon^{4 / 3}}, A(\mathbf{k}, \epsilon=0)=2 \pi \delta\left(\epsilon_{\mathbf{k}}-\mu\right),
$$

and $c=\left(k_{F} / m\right) \chi_{D}^{-2 / 3} k_{0}^{-1 / 3}$ [20-26]. We consider the contribution of $q \ll k_{F}$, expanding $\epsilon_{\mathbf{k}+\mathbf{q}} \approx v_{F}|\mathbf{k}+\mathbf{q}| ;$ we then apply the Sommerfeld expansion according to $\epsilon_{1}$, and the largest contribution at low $T$ comes from the term where the derivative is applied to $n_{F}\left(\epsilon_{1}-\epsilon_{2}+\epsilon_{3}\right)$,

$\kappa_{\mathrm{sp}} \approx J_{\perp}^{\mathrm{sp} 2} \int \frac{d^{2} k d^{2} k^{\prime} d^{2} q}{(2 \pi)^{6}} \int \frac{d \epsilon_{2} d \epsilon_{3}}{(2 \pi)^{2}} A(\mathbf{k}, 0) A\left(\mathbf{k}+\mathbf{q}, \epsilon_{2}\right) A\left(\mathbf{k}^{\prime}, \epsilon_{3}\right) A\left(\mathbf{k}^{\prime}-\mathbf{q},-\epsilon_{2}+\epsilon_{3}\right) \epsilon_{2}^{2} n_{F}\left(\epsilon_{2}\right) n_{F}\left(-\epsilon_{3}\right) \partial_{\epsilon} n_{F}\left(-\epsilon_{2}+\epsilon_{3}\right)$,

which, at low temperatures, becomes

$$
\kappa_{\mathrm{sp}} \approx J_{\perp}^{\mathrm{sp} 2} T \int \frac{d^{2} k d^{2} k^{\prime} d^{2} q}{(2 \pi)^{6}} \int \frac{d \epsilon_{2}}{2 \pi} A(\mathbf{k}, 0) A\left(\mathbf{k}+\mathbf{q}, \epsilon_{2}\right) A\left(\mathbf{k}^{\prime}, \epsilon_{2}\right) A\left(\mathbf{k}^{\prime}-\mathbf{q}, 0\right) \frac{\partial n_{F}\left(\epsilon_{2}\right)}{\partial \epsilon_{2}} \epsilon_{2}^{2}
$$

Using

$$
\int \frac{d^{2} k}{(2 \pi)^{2}} A(\mathbf{k}, 0) A(\mathbf{k}+\mathbf{q}, \epsilon)=\nu \int d \theta \frac{c \epsilon^{2 / 3}}{v_{F}^{2} q^{2} \cos ^{2} \theta+c^{2} \epsilon^{4 / 3}}=\frac{\nu}{\sqrt{v_{F}^{2} q^{2}+c^{2} \epsilon^{4 / 3}}}
$$

we find that

$$
\kappa_{\mathrm{sp}} \approx J_{\perp}^{\mathrm{sp} 2} \nu^{2} T \int \frac{d \epsilon}{2 \pi} \frac{\partial n_{F}(\epsilon)}{\partial \epsilon} \int \frac{d^{2} q}{(2 \pi)^{2}} \frac{\epsilon^{2}}{v_{F}^{2} q^{2}+c^{2} \epsilon^{4 / 3}} \approx \frac{J_{\perp}^{\mathrm{s} 2} \nu^{2}}{v_{F}^{2}} T^{3} \log \left(\frac{T}{(W / c)^{3 / 2}}\right)
$$

where $W$ is a UV cutoff.

\section{Clean gauge photon thermal conductivity}

In the case of a U(1) QSL, the coupling of the interlayer gauge fields is given by Eq. (22),

$$
H_{\perp, \mathrm{ph}}=J_{\perp}^{\mathrm{ph}} \int \frac{d^{2} k}{(2 \pi)^{2}} k^{2} a_{l}^{T}(k) a_{l^{\prime}}^{T}\left(k^{\prime}\right) .
$$

The $c$-axis thermal conductivity in the clean case is

$$
\frac{\left(J_{\perp}^{\mathrm{ph}}\right)^{2}}{T} \int \frac{d^{2} k}{(2 \pi)^{2}} k^{4} \int_{0}^{\infty} d \epsilon A_{\mathrm{ph}}^{2}(\mathbf{k}, \epsilon) \epsilon^{2} \partial_{\epsilon} n_{B}(\epsilon)
$$

where $A_{\mathrm{ph}}(\mathbf{k}, \epsilon)=\gamma\left[|\omega| k /\left(\chi^{2} k^{6}+\gamma^{2} \omega^{2}\right)\right]$ is the photon spectral function. This results in 


$$
\begin{aligned}
\kappa_{c} & =\frac{\gamma^{2}\left(J_{\perp}^{\mathrm{ph}}\right)^{2}}{T} \int \frac{d^{2} k}{(2 \pi)^{2}} k^{6} \int_{0}^{\infty} d \epsilon \frac{\epsilon^{4}}{\left(\chi^{2} k^{6}+\gamma^{2} \epsilon^{2}\right)^{2}} \partial_{\epsilon} n_{B}(\epsilon) \\
& \sim \frac{\gamma^{2}\left(J_{\perp}^{\mathrm{ph}}\right)^{2}}{T}\left(\frac{\gamma}{\chi}\right)^{-4 / 3} \int_{0}^{\infty} d \epsilon \epsilon^{8 / 3} \partial_{\epsilon} n_{B}(\epsilon) \\
& \sim\left(J_{\perp}^{\mathrm{ph}}\right)^{2} \gamma^{2 / 3} \chi^{4 / 3} T^{5 / 3} .
\end{aligned}
$$

In our calculation of the interlayer thermal conductivity, we have neglected processes that transfer a larger number of gauge-invariant excitations between the layers (for example, two spinons and a photon) because their contribution to $\kappa_{c}$ has a higher power of $T$ and is therefore negligible in the limit of low temperature.

[1] P. W. Anderson, Resonating Valence Bonds: A New Kind of Insulator?, Mater. Res. Bull. 8, 153 (1973).

[2] P. W. Anderson, The Resonating Valence Bond State in $\mathrm{La}_{2} \mathrm{CuO}_{4}$ and Superconductivity, Science 235, 1196 (1987).

[3] P. A. Lee, An End to the Drought of Quantum Spin Liquids, Science 321, 1306 (2008).

[4] L. Balents, Spin Liquids in Frustrated Magnets, Nature (London) 464, 199 (2010).

[5] L. Savary and L. Balents, Quantum Spin Liquids: A Review, Rep. Prog. Phys. 80, 016502 (2017).

[6] Y. Zhou, K. Kanoda, and T.-K. Ng, Quantum Spin Liquid States, Rev. Mod. Phys. 89, 025003 (2017).

[7] A. Kitaev and J. Preskill, Topological Entanglement Entropy, Phys. Rev. Lett. 96, 110404 (2006).

[8] M. Levin and X.-G. Wen, Detecting Topological Order in a Ground State Wave Function, Phys. Rev. Lett. 96, 110405 (2006).

[9] S. A. Kivelson, D. S. Rokhsar, and J. P. Sethna, Topology of the Resonating Valence-Bond State: Solitons and High-T Superconductivity, Phys. Rev. B 35, 8865 (1987).

[10] N. Read and S. Sachdev, Large-N Expansion for Frustrated Quantum Antiferromagnets, Phys. Rev. Lett. 66, 1773 (1991).

[11] R. A. Jalabert and S. Sachdev, Spontaneous Alignment of Frustrated Bonds in an Anisotropic, Three-Dimensional Ising Model, Phys. Rev. B 44, 686 (1991).

[12] S. Sachdev, Kagome and Triangular-Lattice Heisenberg Antiferromagnets: Ordering from Quantum Fluctuations and Quantum-Disordered Ground States with Unconfined Bosonic Spinons, Phys. Rev. B 45, 12377 (1992).

[13] T. Senthil and M. P. A. Fisher, $Z_{2}$ Gauge Theory of Electron Fractionalization in Strongly Correlated Systems, Phys. Rev. B 62, 7850 (2000).

[14] R. Moessner and S. L. Sondhi, Resonating Valence Bond Phase in the Triangular Lattice Quantum Dimer Model, Phys. Rev. Lett. 86, 1881 (2001).

[15] R. Moessner, S. L. Sondhi, and E. Fradkin, ShortRanged Resonating Valence Bond Physics, Quantum Dimer Models, and Ising Gauge Theories, Phys. Rev. B 65, 024504 (2001).
[16] A. Kitaev, Anyons in an Exactly Solved Model and Beyond, Ann. Phys. (Amsterdam) 321, 2 (2006), January Special Issue.

[17] M. Barkeshli, H. Yao, and S. A. Kivelson, Gapless Spin Liquids: Stability and Possible Experimental Relevance, Phys. Rev. B 87, 140402 (2013).

[18] C. Nayak and F. Wilczek, Non-Fermi Liquid Fixed Point in $2+1$ Dimensions, Nucl. Phys. B417, 359 (1994).

[19] B. L. Altshuler, L. B. Ioffe, and A. J. Millis, Low-Energy Properties of Fermions with Singular Interactions, Phys. Rev. B 50, 14048 (1994).

[20] O. I. Motrunich and T. Senthil, Exotic Order in Simple Models of Bosonic Systems, Phys. Rev. Lett. 89, 277004 (2002).

[21] T. Senthil and O. Motrunich, Microscopic Models for Fractionalized Phases in Strongly Correlated Systems, Phys. Rev. B 66, 205104 (2002).

[22] P. A. Lee, N. Nagaosa, and X.-G. Wen, Doping a Mott Insulator: Physics of High-Temperature Superconductivity, Rev. Mod. Phys. 78, 17 (2006).

[23] L. B. Ioffe and A. I. Larkin, Gapless Fermions and Gauge Fields in Dielectrics, Phys. Rev. B 39, 8988 (1989).

[24] N. Nagaosa and P. A. Lee, Normal-State Properties of the Uniform Resonating-Valence-Bond State, Phys. Rev. Lett. 64, 2450 (1990).

[25] P. A. Lee and N. Nagaosa, Gauge Theory of the Normal State of High- $t_{c}$ Superconductors, Phys. Rev. B 46, 5621 (1992).

[26] J. Polchinski, Low-Energy Dynamics of the Spinon-Gauge System, Nucl. Phys. B422, 617 (1994).

[27] G. Jackeli and G. Khaliullin, Mott Insulators in the Strong Spin-Orbit Coupling Limit: From Heisenberg to a Quantum Compass and Kitaev Models, Phys. Rev. Lett. 102, 017205 (2009).

[28] J. Chaloupka, G. Jackeli, and G. Khaliullin, KitaevHeisenberg Model on a Honeycomb Lattice: Possible Exotic Phases in Iridium Oxides $\mathrm{A}_{2} \mathrm{IrO}_{3}$, Phys. Rev. Lett. 105, 027204 (2010).

[29] X. Liu, T. Berlijn, W.-G. Yin, W. Ku, A. Tsvelik, Y.-J. Kim, H. Gretarsson, Y. Singh, P. Gegenwart, and J. P. Hill, LongRange Magnetic Ordering in $\mathrm{Na}_{2} \mathrm{IrO}_{3}$, Phys. Rev. B 83, 220403 (2011).

[30] S. K. Choi, R. Coldea, A. N. Kolmogorov, T. Lancaster, I. I. Mazin, S. J. Blundell, P. G. Radaelli, Y. Singh, P. Gegenwart, K. R. Choi, S.-W. Cheong, P. J. Baker, C. Stock, and J. Taylor, Spin Waves and Revised Crystal Structure of Honeycomb Iridate $\mathrm{Na}_{2} \mathrm{IrO}_{3}$, Phys. Rev. Lett. 108, 127204 (2012).

[31] M. Yamashita, N. Nakata, Y. Senshu, M. Nagata, H. M. Yamamoto, R. Kato, T. Shibauchi, and Y. Matsuda, Highly Mobile Gapless Excitations in a Two-Dimensional Candidate Quantum Spin Liquid, Science 328, 1246 (2010).

[32] S. Yamashita, Y. Nakazawa, M. Oguni, Y. Oshima, H. Nojiri, Y. Shimizu, K. Miyagawa, and K. Kanoda, Thermodynamic Properties of a Spin-1/2 Spin-Liquid State in a Kappa-type Organic Salt, Nat. Phys. 4, 459 (2008).

[33] S. Yamashita, T. Yamamoto, Y. Nakazawa, M. Tamura, and R. Kato, Gapless Spin Liquid of an Organic Triangular Compound Evidenced by Thermodynamic Measurements, Nat. Commun. 2, 275 (2011). 
[34] M. Yamashita, N. Nakata, Y. Kasahara, T. Sasaki, N. Yoneyama, N. Kobayashi, S. Fujimoto, T. Shibauchi, and Y. Matsuda, Thermal-Transport Measurements in a Quantum Spin-Liquid State of the Frustrated Triangular Magnet(BEDT-TTF $)_{2} \mathrm{Cu}_{2}(\mathrm{CN})_{3}$, Nat. Phys. 5, 44 (2009).

[35] P. Mendels and F. Bert, Quantum Kagome Antiferromagnet $\mathrm{ZnCu}_{3}(\mathrm{OH})_{6} \mathrm{Cl}_{2}$, J. Phys. Soc. Jpn. 79, 011001 (2010).

[36] M. P. Shores, E. A. Nytko, B. M. Bartlett, and D. G. Nocera, A Structurally Perfect $s=1 / 2$ Kagomé Antiferromagnet, J. Am. Chem. Soc. 127, 13462 (2005).

[37] H. O. Jeschke, F. Salvat-Pujol, and R. Valentí, FirstPrinciples Determination of Heisenberg Hamiltonian Parameters for the Spin- $\frac{1}{2}$ Kagome Antiferromagnet $\mathrm{ZnCu}_{3}(\mathrm{OH})_{6} \mathrm{Cl}_{2}$, Phys. Rev. B 88, 075106 (2013).

[38] A. Olariu, P. Mendels, F. Bert, F. Duc, J. C. Trombe, M. A. de Vries, and A. Harrison, ${ }^{17} \mathrm{O} N M R$ Study of the Intrinsic Magnetic Susceptibility and Spin Dynamics of the Quantum Kagome Antiferromagnet $\mathrm{ZnCu}_{3}(\mathrm{OH})_{6} \mathrm{Cl}_{2}$, Phys. Rev. Lett. 100, 087202 (2008).

[39] J. S. Helton, K. Matan, M. P. Shores, E. A. Nytko, B. M. Bartlett, Y. Qiu, D. G. Nocera, and Y. S. Lee, Dynamic Scaling in the Susceptibility of the Spin- $\frac{1}{2}$ Kagome Lattice Antiferromagnet Herbertsmithite, Phys. Rev. Lett. 104, 147201 (2010).

[40] J. S. Helton, K. Matan, M. P. Shores, E. A. Nytko, B. M. Bartlett, Y. Yoshida, Y. Takano, A. Suslov, Y. Qiu, J.-H. Chung, D. G. Nocera, and Y. S. Lee, Spin Dynamics of the Spin-1/2 Kagome Lattice Antiferromagnet $\mathrm{ZnCu}_{3}(\mathrm{OH})_{6} \mathrm{Cl}_{2}$, Phys. Rev. Lett. 98, 107204 (2007).

[41] M. A. de Vries, J. R. Stewart, P. P. Deen, J. O. Piatek, G. J. Nilsen, H. M. Rønnow, and A. Harrison, Scale-Free Antiferromagnetic Fluctuations in the $s=1 / 2$ Kagome Antiferromagnet Herbertsmithite, Phys. Rev. Lett. 103, 237201 (2009).

[42] F. Bert, S. Nakamae, F. Ladieu, D. L'Hôte, P. Bonville, F. Duc, J.-C. Trombe, and P. Mendels, Low Temperature Magnetization of the $s=\frac{1}{2}$ Kagome Antiferromagnet $\mathrm{ZnCu}_{3}(\mathrm{OH})_{6} \mathrm{Cl}_{2}$, Phys. Rev. B 76, 132411 (2007).

[43] T.-H. Han, J. S. Helton, S. Chu, D. G. Nocera, J. A. Rodriguez-Rivera, C. Broholm, and Y. S. Lee, Fractionalized Excitations in the Spin-Liquid State of a KagomeLattice Antiferromagnet, Nature (London) 492, 406 (2012).

[44] D. V. Pilon, C. H. Lui, T. H. Han, D. Shrekenhamer, A. J. Frenzel, W. J. Padilla, Y. S. Lee, and N. Gedik, Spin-Induced Optical Conductivity in the Spin-Liquid Candidate Herbertsmithite, Phys. Rev. Lett. 111, 127401 (2013).

[45] T.-H. Han, R. Chisnell, C. J. Bonnoit, D. E. Freedman, V. S. Zapf, N. Harrison, D. G. Nocera, Y. Takano, and Y. S. Lee, Thermodynamic Properties of the Quantum Spin Liquid Candidate $\mathrm{ZnCu}_{3}(\mathrm{OH})_{6} \mathrm{Cl}_{2}$ in High Magnetic Fields, arXiv:1402.2693.

[46] M. Fu, T. Imai, T.-H. Han, and Y. S. Lee, Evidence for a Gapped Spin-Liquid Ground State in a Kagome Heisenberg Antiferromagnet, Science 350, 655 (2015).

[47] Z. Zou and P. W. Anderson, Neutral Fermion, Charge-e Boson Excitations in the Resonating-Valence-Bond State and Superconductivity in $\mathrm{La}_{2} \mathrm{CuO}_{4}$-Based Compounds, Phys. Rev. B 37, 627 (1988).

[48] S. Kivelson, Statistics of Holons in the Quantum Hard-Core Dimer Gas, Phys. Rev. B 39, 259 (1989).
[49] D. Arovas, J. R. Schrieffer, and F. Wilczek, Fractional Statistics and the Quantum Hall Effect, Phys. Rev. Lett. 53, 722 (1984).

[50] N. Read and B. Chakraborty, Statistics of the Excitations of the Resonating-Valence-Bond State, Phys. Rev. B 40, 7133 (1989).

[51] T. Senthil and M. P. A. Fisher, Fractionalization in the Cuprates: Detecting the Topological Order, Phys. Rev. Lett. 86, 292 (2001).

[52] T. Senthil and M.P. A. Fisher, Detecting Fractions of Electrons in the High- $T_{c}$ Cuprates, Phys. Rev. B 64, 214511 (2001).

[53] M. R. Norman and T. Micklitz, How to Measure a Spinon Fermi Surface, Phys. Rev. Lett. 102, 067204 (2009).

[54] M. Barkeshli, E. Berg, and S. Kivelson, Coherent Transmutation of Electrons into Fractionalized Anyons, Science 346, 722 (2014).

[55] S. Chatterjee and S. Sachdev, Probing Excitations in Insulators via Injection of Spin Currents, Phys. Rev. B 92, 165113 (2015).

[56] J. Nasu, J. Knolle, D. L. Kovrizhin, Y. Motome, and R. Moessner, Fermionic Response from Fractionalization in an Insulating Two-Dimensional Magnet, Nat. Phys. 12, 912 (2016).

[57] S. C. Morampudi, A. M. Turner, F. Pollmann, and F. Wilczek, Statistics of Fractionalized Excitations through Threshold Spectroscopy, Phys. Rev. Lett. 118, 227201 (2017).

[58] R. Coldea, D. A. Tennant, and Z. Tylczynski, Extended Scattering Continua Characteristic of Spin Fractionalization in the Two-Dimensional Frustrated Quantum Magnet $\mathrm{Cs}_{2} \mathrm{CuCl}_{4}$ Observed by Neutron Scattering, Phys. Rev. B 68, 134424 (2003).

[59] M. Punk, D. Chowdhury, and S. Sachdev, Topological Excitations and the Dynamic Structure Factor of Spin Liquids on the Kagome Lattice, Nat. Phys. 10, 289 (2014).

[60] Here, we assume that the interlayer coupling does not destabilize the layered QSL phase. This is certainly the case for a $Z_{2}$ QSL with a Dirac spectrum since the interlayer coupling is irrelevant. For the case of a QSL with a Fermi surface, the interlayer coupling is marginal at tree level; we assume that we are at temperatures above the (exponentially small) temperature of any possible instability.

[61] A similar mechanism can provide evidence for fractionalization in the $c$-axis electrical transport in a metallic resonating valence bond state. See P. W. Anderson and Z. Zou, "Normal" Tunneling and "Normal" Transport: Diagnostics for the Resonating-Valence-Bond State, Phys. Rev. Lett. 60, 132 (1988); N. Nagaosa, Interlayer charge dynamics of the uniform resonating-valence-bond state, Phys. Rev. B 52, 10561 (1995).

[62] A. C. Durst and P. A. Lee, Impurity-Induced Quasiparticle Transport and Universal-Limit Wiedemann-Franz Violation in d-Wave Superconductors, Phys. Rev. B 62, 1270 (2000).

[63] C. P. Nave and P. A. Lee, Transport Properties of a Spinon Fermi Surface Coupled to a U(1) Gauge Field, Phys. Rev. B 76, 235124 (2007).

[64] S. Yang, D. L. Zhou, and C. P. Sun, Mosaic Spin Models with Topological Order, Phys. Rev. B 76, 180404 (2007). 
[65] G. Baskaran, G. Santhosh, and R. Shankar, Exact Quantum Spin Liquids with Fermi Surfaces in Spin-Half Models, arXiv:0908.1614.

[66] H.-H. Lai and O. I. Motrunich, SU(2)-Invariant Majorana Spin Liquid with Stable Parton Fermi Surfaces in an Exactly Solvable Model, Phys. Rev. B 84, 085141 (2011).

[67] M. Hermanns and S. Trebst, Quantum Spin Liquid with a Majorana Fermi Surface on the Three-Dimensional Hyperoctagon Lattice, Phys. Rev. B 89, 235102 (2014).

[68] M. Hermanns, S. Trebst, and A. Rosch, Spin-Peierls Instability of Three-Dimensional Spin Liquids with Majorana Fermi Surfaces, Phys. Rev. Lett. 115, 177205 (2015).

[69] K. O'Brien, M. Hermanns, and S. Trebst, Classification of Gapless $\mathbb{Z}_{2}$ Spin Liquids in Three-Dimensional Kitaev Models, Phys. Rev. B 93, 085101 (2016).

[70] G. F. Giuliani and J. J. Quinn, Lifetime of a Quasiparticle in a Two-Dimensional Electron Gas, Phys. Rev. B 26, 4421 (1982).

[71] S. Sachdev, Quantum Phase Transitions, 2nd ed. (Cambridge University Press, Cambridge, England, 2011).

[72] A. J. Willans, J. T. Chalker, and R. Moessner, Disorder in a Quantum Spin Liquid: Flux Binding and Local Moment Formation, Phys. Rev. Lett. 104, 237203 (2010).

[73] A. W. W. Ludwig, M. P. A. Fisher, R. Shankar, and G. Grinstein, Integer Quantum Hall Transition: An Alternative Approach and Exact Results, Phys. Rev. B 50, 7526 (1994).

[74] J. M. Luttinger, Theory of Thermal Transport Coefficients, Phys. Rev. 135, A1505 (1964).

[75] J. M. Luttinger, Thermal Transport Coefficients of a Superconductor, Phys. Rev. 136, A1481 (1964).

[76] B.S. Shastry, Electrothermal Transport Coefficients at Finite Frequencies, Rep. Prog. Phys. 72, 016501 (2009).

[77] Here, we neglect weak localization corrections, which are beyond the Born approximation. We assume that we are at not-too-low temperatures, such that weak localization effects are unimportant.

[78] A. Altland and B. Simons, Condensed Matter Field Theory, 2nd ed. (Cambridge University Press, Cambridge, England, 2010).

[79] S.-S. Lee, Low-Energy Effective Theory of Fermi Surface Coupled with U(1) Gauge Field in $2+1$ Dimensions, Phys. Rev. B 80, 165102 (2009).

[80] D. F. Mross, J. McGreevy, H. Liu, and T. Senthil, Controlled Expansion for Certain Non-Fermi-Liquid Metals, Phys. Rev. B 82, 045121 (2010).
[81] D. Dalidovich and S.-S. Lee, Perturbative Non-Fermi Liquids from Dimensional Regularization, Phys. Rev. B 88, 245106 (2013).

[82] V. M. Galitski, Metallic Phase in a Two-Dimensional Disordered Fermi System with Singular Interactions, Phys. Rev. B 72, 214201 (2005).

[83] B. Fåk, E. Kermarrec, L. Messio, B. Bernu, C. Lhuillier, F. Bert, P. Mendels, B. Koteswararao, F. Bouquet, J. Ollivier, A. D. Hillier, A. Amato, R. H. Colman, and A. S. Wills, Kapellasite: A Kagome Quantum Spin Liquid with Competing Interactions, Phys. Rev. Lett. 109, 037208 (2012).

[84] H. O. Jeschke, F. Salvat-Pujol, and R. Valentí, FirstPrinciples Determination of Heisenberg Hamiltonian Parameters for the Spin- $\frac{1}{2}$ Kagome Antiferromagnet $\mathrm{ZnCu}_{3}(\mathrm{OH})_{6} \mathrm{Cl}_{2}$, Phys. Rev. B 88, 075106 (2013).

[85] T.-H. Han, M. R. Norman, J.-J. Wen, J. A. RodriguezRivera, J. S. Helton, C. Broholm, and Y. S. Lee, Correlated Impurities and Intrinsic Spin-Liquid Physics in the Kagome Material Herbertsmithite, Phys. Rev. B 94, 060409(R) (2016).

[86] K. Slagle, W. Choi, L. E. Chern, and Y. B. Kim, Theory of a Quantum Spin Liquid in Hydrogen-Intercalated Honeycomb Iridate, $\mathrm{H}_{3} \operatorname{LiIr}_{2} \mathrm{O}_{6}$, Phys. Rev. B 97, 115159 (2018).

[87] A. Banerjee, C. A. Bridges, J.-Q. Yan, A. A. Aczel, L. Li, M. B. Stone, G. E. Granroth, M. D. Lumsden, Y. Yiu, J. Knolle et al., Proximate Kitaev Quantum Spin Liquid Behaviour in a Honeycomb Magnet, Nat. Mater. 15, 733 (2016).

[88] P. Lampen-Kelley, A. Banerjee, A. A. Aczel, H. B. Cao, J.-Q. Yan, S. E. Nagler, and D. Mandrus, Destabilization of Magnetic Order in a Dilute Kitaev Spin Liquid Candidate, Phys. Rev. Lett. 119, 237203 (2017).

[89] M. Yamashita, private communication.

[90] E. H. Lieb, Flux Phase of the Half-Filled Band, Phys. Rev. Lett. 73, 2158 (1994).

[91] E. K.-H. Lee and Y. B. Kim, Theory of Magnetic Phase Diagrams in Hyperhoneycomb and Harmonic-Honeycomb Iridates, Phys. Rev. B 91, 064407 (2015).

[92] The factor of $T$ in front of $\chi$ in Eq. (C14) comes our convention of the Matsubara fermionic fields: $\psi\left(i \nu_{n}\right)=$ $T^{1 / 2} \int_{0}^{\beta} d \tau e^{i \nu_{n} \tau} \psi(\tau)$. In this convention, there are no factors of $T$ in the quadratic part of the action.

[93] G. D. Mahan, Many-Particle Physics, 3rd ed. (Plenum, New York, N.Y., 1993). 